Pacific

Journal of

Mathematics

\title{
ON NONLINEAR NONHOMOGENEOUS RESONANT DIRICHLET EQUATIONS
}

\author{
Nikolaos S. Papageorgiou AND GeORge SMyRlis
}




\title{
ON NONLINEAR NONHOMOGENEOUS RESONANT DIRICHLET EQUATIONS
}

\author{
Nikolaos S. Papageorgiou and George Smyrlis
}

We consider a $(p, 2)$-equation with a Carathéodory reaction $f(z, x)$ which is resonant at $\pm \infty$ and has constant sign, $z$-dependent zeros. Using variational methods, together with truncation and comparison techniques and Morse theory, we establish the existence of five nontrivial smooth solutions (four of constant sign and the fifth nodal). If the reaction $f(z, x)$ is $C^{\mathbf{1}}$ in $x \in \mathbb{R}$, then we produce a second nodal solution for a total of six nontrivial smooth solutions.

\section{Introduction}

Let $\Omega \subseteq \mathbb{R}^{N}$ be a bounded domain with a $C^{2}$-boundary $\partial \Omega$. In this paper we study the nonlinear Dirichlet problem

$$
-\Delta_{p} u(z)-\Delta u(z)=f(z, u(z)) \quad \text { in } \Omega,\left.\quad u\right|_{\partial \Omega}=0, \quad 2<p .
$$

Here $\Delta_{p}$ denotes the $p$-Laplacian differential operator defined by

$$
\Delta_{p} u(z)=\operatorname{div}\left(\|D u(z)\|^{p-2} D u(z)\right) \quad \text { for all } u \in W_{0}^{1, p}(\Omega) .
$$

Problem (1) is important in quantum physics in connection with Derrick's model [Derrick 1964] for the existence of solitons, which was investigated in more detail by Benci, D'Avenia, Fortunato, and Pisani [Benci et al. 2000]. Recently, such equations attracted the interest of people working on nonlinear partial differential equations and some existence and multiplicity results were proved in [Cingolani and Degiovanni 2005; Cingolani and Vannella 2003; Sun 2012]. All consider nonresonant equations. In contrast, in this work we deal with the resonant case. More precisely, we assume that, asymptotically at $\pm \infty$, we have resonance with respect to the first eigenvalue of $\left(-\Delta_{p}, W_{0}^{1, p}(\Omega)\right)$. In problem (1) the reaction $f(z, x)$ is a Carathéodory function (i.e., for all $x \in \mathbb{R}, z \rightarrow f(z, x)$ is measurable, and, for a.a. $z \in \Omega, x \rightarrow f(z, x)$ is continuous) and has positive and negative zeros which in general depend on $z \in \Omega$. Problems driven by the $p$-Laplacian, and with a

MSC2010: 35J20, 35J60, 35J92, 58E05.

Keywords: resonant equations, tangency principle, strong comparison principle, constant sign and nodal solutions, Morse theory. 
reaction that has zeros, were studied by Bartsch, Liu, and Weth [Bartsch et al. 2005] (they assume that the zeros are constant) and by Iturriaga, Massa, Sánchez, and Ubilla [Iturriaga et al. 2010] (they have variable zeros). In both works the reaction $f(z, \cdot)$ is $(p-1)$-superlinear.

Here, we prove the existence of at least five nontrivial smooth solutions and provide sign information for all of them (two are positive, two are negative and the fifth is nodal). Moreover, by strengthening the regularity of $f(z, \cdot)$ (namely, assuming that $f(z, \cdot) \in C^{1}(\mathbb{R})$ ), we produce a second nodal solution for a total of six nontrivial smooth solutions, all with precise sign information.

Our approach is variational based on the critical point theory, coupled with suitable truncation and comparison techniques and with Morse theory (critical groups). In the next section, for the convenience of the reader, we recall the main mathematical tools that we will use in this work.

\section{Mathematical background}

Let $X$ be a Banach space. By $X^{*}$ we denote the topological dual of $X$ and by $\langle\cdot, \cdot\rangle$ the duality brackets for the pair $\left(X^{*}, X\right)$. Let $\varphi \in C^{1}(X)$. We say that $\varphi$ satisfies the Cerami condition if the following is true:

C-condition. Every sequence $\left\{x_{n}\right\}_{n \geq 1} \subseteq X$ such that $\left\{\varphi\left(x_{n}\right)\right\}_{n \geq 1} \subseteq \mathbb{R}$ is bounded and

$$
\left(1+\left\|x_{n}\right\|\right) \varphi^{\prime}\left(x_{n}\right) \rightarrow 0 \quad \text { in } X^{*} \text { as } n \rightarrow \infty
$$

admits a strongly convergent subsequence.

This compactness-type condition is in general weaker than the usual Palais-Smale condition ("PS-condition" for short). However, it suffices to have a deformation theorem and from it derive the minimax theory of certain critical values of $\varphi$ (see, for example, [Gasiński and Papageorgiou 2006]). In particular, we can state the following theorem, known in the literature as the mountain pass theorem [ibid., p. 648].

Theorem 1. If $\varphi \in C^{1}(X)$ satisfies the $C$-condition, $x_{0}, x_{1} \in X, \rho>0,\left\|x_{0}-x_{1}\right\|>\rho$,

$$
\max \left\{\varphi\left(x_{0}\right), \varphi\left(x_{1}\right)\right\}<\inf \left[\varphi(x):\left\|x-x_{0}\right\|=\rho\right]=\eta_{\rho}
$$

and

$$
c=\inf _{\gamma \in \Gamma} \max _{0 \leq t \leq 1} \varphi(\gamma(t)), \quad \text { where } \Gamma=\left\{\gamma \in C([0,1], X): \gamma(0)=x_{0}, \gamma(1)=x_{1}\right\},
$$

then $c \geq \eta_{\rho}$ and $c$ is a critical value of $\varphi$. 
In the analysis of problem (1), in addition to the Sobolev spaces

$$
W_{0}^{1, p}(\Omega), \quad H_{0}^{1}(\Omega)
$$

we will also use the Banach space

$$
C_{0}^{1}(\bar{\Omega})=\left\{u \in C^{1}(\bar{\Omega}):\left.u\right|_{\partial \Omega}=0\right\} .
$$

This is an ordered Banach space with positive cone

$$
C_{+}=\left\{u \in C_{0}^{1}(\bar{\Omega}): u(z) \geq 0 \text { for all } z \in \Omega\right\} .
$$

This cone has a nonempty interior given by

$$
\text { int } C_{+}=\left\{u \in C_{+}: u(z)>0 \text { for all } z \in \Omega, \frac{\partial u}{\partial n}(z)<0 \text { for all } z \in \partial \Omega\right\}
$$

(here $n(\cdot)$ denotes the outward unit normal on $\partial \Omega$ ).

Let $f_{0}: \Omega \times \mathbb{R} \rightarrow \mathbb{R}$ be a Carathéodory function with subcritical growth in $x \in \mathbb{R}$; i.e.,

$$
\left|f_{0}(z, x)\right| \leq \hat{a}(z)+\hat{c}|x|^{r-1} \quad \text { for a.a. } z \in \Omega \text {, all } x \in \mathbb{R},
$$

with $\hat{a} \in L^{\infty}(\Omega)_{+}, \hat{c}>0$, and

$$
1<r<p^{*}=\left\{\begin{array}{cl}
\frac{N p}{N-p} & \text { if } p<N, \\
+\infty & \text { if } p \geq N .
\end{array}\right.
$$

We set $F_{0}(z, x)=\int_{0}^{x} f_{0}(z, s) d s$ and consider the $C^{1}$-functional $\varphi_{0}: W_{0}^{1, p}(\Omega) \rightarrow$ $\mathbb{R}$ defined by

$$
\varphi_{0}(u)=\frac{1}{p}\|D u\|_{p}^{p}+\frac{1}{2}\|D u\|_{2}^{2}-\int_{\Omega} F_{0}(z, u(z)) d z \quad \text { for all } u \in W_{0}^{1, p}(\Omega) .
$$

The next theorem is a particular case of a more general result of [Gasiński and Papageorgiou 2012].

Theorem 2. If $u_{0} \in W_{0}^{1, p}(\Omega)$ is a local $C_{0}^{1}(\bar{\Omega})$-minimizer of $\varphi_{0}$, i.e., there exists $\rho_{0}>0$ such that

$$
\varphi_{0}\left(u_{0}\right) \leq \varphi_{0}\left(u_{0}+h\right) \quad \text { for all } h \in C_{0}^{1}(\bar{\Omega}) \text { with }\|h\|_{C_{0}^{1}(\bar{\Omega})} \leq \rho_{0},
$$

then $u_{0} \in C^{1, \alpha}(\bar{\Omega})$ for some $\alpha \in(0,1)$ and $u_{0}$ is also a local $W_{0}^{1, p}(\Omega)$-minimizer of $\varphi_{0}$; i.e., there exists $\rho_{1}>0$ such that

$$
\varphi_{0}\left(u_{0}\right) \leq \varphi_{0}\left(u_{0}+h\right) \quad \text { for all } h \in W_{0}^{1, p}(\Omega) \text { with }\|h\| \leq \rho_{1} .
$$


Remark. We should mention that the first such result was proved by Brézis and Nirenberg [1993] and was later extended by García Azorero, Peral Alonso, and Manfredi [García Azorero et al. 2000].

Let $h, \hat{h} \in L^{\infty}(\Omega)$. We write $h \prec \hat{h}$ if, for every compact $K \subseteq \Omega$, we can find $\varepsilon>0$ such that

$$
h(z)+\varepsilon \leq \hat{h}(z) \text { for a.a. } z \in K .
$$

Clearly, if $h, \hat{h} \in C(\Omega)$ and $h(z)<\hat{h}(z)$ for all $z \in \Omega$, then $h \prec \hat{h}$. A straightforward modification of the proof of Proposition 2.6 of [Arcoya and Ruiz 2006] in order to accommodate the extra linear term $-\Delta u$ gives the following strong comparison principle.

Proposition 3. If $\xi \geq 0, h, \hat{h} \in L^{\infty}(\Omega), h \prec \hat{h}, u, v \in C_{0}^{1}(\bar{\Omega})$ are solutions of

$$
\begin{aligned}
& -\Delta_{p} u(z)-\Delta u(z)+\xi|u(z)|^{p-2} u(z)=h(z), \\
& -\Delta_{p} v(z)-\Delta v(z)+\xi|v(z)|^{p-2} v(z)=\hat{h}(z) \quad \text { in } \Omega,
\end{aligned}
$$

and $v \in \operatorname{int} C_{+}$, then $v-u \in \operatorname{int} C_{+}$.

Proof. We follow [Arcoya and Ruiz 2006] (see Proposition 2.6).

By nonlinear regularity, $u, v \in C^{1, \beta}(\bar{\Omega})(0<\beta<1)$.

We have

$A_{p}(u)+A(u)+\xi|u|^{p-2} u=h \leq \hat{h}=A_{p}(v)+A(v)+\xi v^{p-1} \quad$ in $W^{-1, p^{\prime}}(\Omega)$.

Acting with $(u-v)^{+} \in W_{0}^{1, p}(\Omega)$, we obtain

$$
\begin{aligned}
\left\langle A_{p}(u)-A_{p}(v),(u-v)^{+}\right\rangle+\left\langle A(u)-A(v),(u-v)^{+}\right\rangle & \\
& +\int_{\Omega} \xi\left(|u|^{p-2} u-v^{p-1}\right)(u-v)^{+} d z \leq 0,
\end{aligned}
$$

which implies that $\left\|D(u-v)^{+}\right\|_{2}^{2} \leq 0$, since $A_{p}$ is monotone; hence $u \leq v$.

First we show that $u(z) \leq v(z)$ for all $z \in \Omega$. For this purpose, we introduce

$$
D_{0}=\{z \in \Omega: u(z)=v(z)\} \quad \text { and } \quad D_{1}=\{z \in \Omega: D u(z)=D v(z)=0\} .
$$

We show that $D_{0} \subseteq D_{1}$. So, let $z_{0} \in D_{0}$. Since $u \leq v$, the function $z \mapsto(u-v)(z)$ attains its maximum at $z_{0} \in D_{0}$ and so we have $D u\left(z_{0}\right)=D v\left(z_{0}\right)$. If $D u\left(z_{0}\right) \neq 0$, then we can find $\bar{B}_{\rho}\left(z_{0}\right) \subseteq \Omega$ such that

$$
\|D u(z)\|>0, \quad\|D v(z)\|>0, \quad(D u(z), D v(z))_{\mathbb{R}^{N}}>0 \quad \text { for all } z \in \bar{B}_{\rho}\left(z_{0}\right) .
$$

We set $w=v-u \in C_{+} \backslash\{0\}$. Then $w$ satisfies the linear elliptic equation

$$
-\sum_{i, j=1}^{N} \frac{\partial}{\partial z_{i}}\left(\eta_{i j}(z) \frac{\partial w}{\partial z_{j}}\right)=-\xi\left(v^{p-1}-|u|^{p-2} u\right)+\hat{h}-h .
$$


In this equation the coefficients $\eta_{i j}(\cdot)$ are given by

$$
\eta_{i j}(z)=\delta_{i j}\left(\|D u(z)\|^{p-2}+1\right)+(p-2)\|D u(z)\|^{p-4} \frac{\partial u}{\partial z_{i}}(z) \frac{\partial u}{\partial z_{j}}(z)
$$

for all $z \in \bar{B}_{\rho}\left(z_{0}\right)$ (see [Arcoya and Ruiz 2006, p. 854]). Hence $\eta_{i j} \in C^{\beta}\left(\bar{B}_{\rho}\left(z_{0}\right)\right.$ ) with $\beta \in(0,1)$ and the $\eta_{i j}$ form a uniformly elliptic operator by taking $\rho \in(0,1)$ even smaller if necessary. Then the strong maximum principle (see [Gilbarg and Trudinger 2001; Vázquez 1984]) implies that

$$
u(z)<v(z) \text { for all } z \in \bar{B}_{\rho}\left(z_{0}\right),
$$

which contradicts the fact that $z_{0} \in D_{0}$. So, we infer that $D_{0} \subseteq D_{1}$.

Since by hypothesis $v \in \operatorname{int} C_{+}$, we see that $D_{1}$ is compact and so $D_{0}$ is compact. So, we can find $\Omega_{1} \subseteq \Omega$ open and smooth such that

$$
D_{0} \subseteq \Omega_{1} \subseteq \bar{\Omega}_{1} \subseteq \Omega
$$

We can find $\varepsilon>0$ such that

$$
\begin{array}{ll}
u(z)+\varepsilon<v(z) & \text { for all } z \in \partial \Omega_{1}, \\
h(z)+\varepsilon<\hat{h}(z) & \text { for a.a. } z \in \Omega_{1} .
\end{array}
$$

Let $\delta \in(0, \min \{\varepsilon, 1\})$ be such that $\left.\xi|| s\right|^{p-2} s-\left|s^{\prime}\right|^{p-2} s^{\prime} \mid<\varepsilon \quad$ for all $s, s^{\prime} \in\left[-\|u\|_{\infty},\|v\|_{\infty}\right]$ with $\left|s-s^{\prime}\right|<2 \delta$.

Then we have

$$
\begin{aligned}
-\Delta_{p}(u+\delta)-\Delta(u+\delta)+\xi|u+\delta|^{p-2}(u+\delta) & =-\Delta_{p}(u)-\Delta(u)+\xi|u+\delta|^{p-2}(u+\delta) \\
& =\xi\left[|u+\delta|^{p-2}(u+\delta)-|u|^{p-2} u\right]+h \\
& \leq h+\varepsilon \leq \hat{h}=-\Delta_{p} v-\Delta v+\xi v^{p-1},
\end{aligned}
$$

which implies $u+\delta \leq v$ in $\Omega_{1}$, by the weak maximum principle.

Since $D_{0} \subseteq \Omega_{1}$, we infer that the boundary point theorem is valid for uniformly elliptic operators with Hölder continuous coefficients (see [Finn and Gilbarg 1957, Lemma 7, p. 31; Gilbarg and Trudinger 2001, p. 46]). So, for every $z_{0} \in \partial \Omega$, we have

$$
\frac{\partial w}{\partial n}\left(z_{0}\right)<0
$$

and therefore $v-u \in \operatorname{int} C_{+}$.

We now recall some basic facts concerning the spectrum of $\left(-\Delta_{p}, W_{0}^{1, p}(\Omega)\right)$. We consider the nonlinear eigenvalue problem

$$
\left\{\begin{array}{c}
-\Delta_{p} u(z)=\hat{\lambda}|u(z)|^{p-2} u(z) \quad \text { a.e. in } \Omega \\
\left.u\right|_{\partial \Omega}=0 .
\end{array}\right.
$$


A number $\hat{\lambda} \in \mathbb{R}$ is an eigenvalue of $\left(-\Delta_{p}, W_{0}^{1, p}(\Omega)\right)$ if the problem (3) has a nontrivial solution $\hat{u} \in W_{0}^{1, p}(\Omega)$; that solution is an eigenfunction corresponding to the eigenvalue $\hat{\lambda}$. The smallest eigenvalue $\hat{\lambda}_{1}(p)$ of $\left(-\Delta_{p}, W_{0}^{1, p}(\Omega)\right)$ has the following properties (see [Anane 1987; Anane and Tsouli 1996; García Azorero and Peral Alonso 1987]):

- $\hat{\lambda}_{1}(p)$ is positive and isolated.

- $\hat{\lambda}_{1}(p)$ is simple (its eigenspace is one-dimensional).

$$
\hat{\lambda}_{1}(p)=\inf \left[\frac{\|D u\|_{p}^{p}}{\|u\|_{p}^{p}}: u \in W_{0}^{1, p}(\Omega), u \neq \equiv 0\right] .
$$

In this variational characterization of $\hat{\lambda}_{1}(p)$, the infimum is realized on the corresponding one-dimensional eigenspace. Moreover, it is clear from the third property above that the elements of the one-dimensional eigenspace do not change sign. In the sequel, by $\hat{u}_{1, p} \in W_{0}^{1, p}(\Omega)$, we denote the $L^{p}$-normalized (i.e., $\left\|\hat{u}_{1, p}\right\|_{p}=1$ ) positive eigenfunction corresponding to the eigenvalue $\hat{\lambda}_{1}(p)>0$. The nonlinear regularity theory (see, for example, [Gasiński and Papageorgiou 2006, pp. 737-738]), implies that $\hat{u}_{1, p} \in C_{+} \backslash\{0\}$. Then the nonlinear maximum principle of [Vázquez 1984] says that $\hat{u}_{1, p} \in \operatorname{int} C_{+}$. Since the spectrum $\sigma(p)$ of $\left(-\Delta_{p}, W_{0}^{1, p}(\Omega)\right)$ is closed and $\hat{\lambda}_{1}(p)>0$ is isolated, the second eigenvalue $\hat{\lambda}_{2}(p)=\inf \left[\lambda \in \sigma(p): \lambda>\hat{\lambda}_{1}(p)\right]$ is also well-defined.

If $N=1$ (ordinary differential equation), then $\sigma(p)=\left\{\hat{\lambda}_{k}(p)\right\}_{k \geq 1} \subseteq(0,+\infty)$, where each $\hat{\lambda}_{k}(p)$ is a simple eigenvalue, $\hat{\lambda}_{k}(p) \rightarrow+\infty$ as $k \rightarrow+\infty$ and the corresponding eigenfunctions $\left\{\hat{u}_{k, p}\right\}_{k \geq 1}$ have exactly $k-1$ zeros (see, for example, [Gasiński and Papageorgiou 2006, p. 761]).

If $N \geq 2$ (partial differential equation), then the Ljusternik-Schnirelmann minimax scheme via the Krasnoselskii genus gives us a whole strictly increasing sequence of eigenvalues $\left\{\hat{\lambda}_{k}(p)\right\}_{k \geq 1}$ such that $\hat{\lambda}_{k}(p) \rightarrow+\infty$ as $k \rightarrow+\infty$. It is not known if this is the complete list of eigenvalues. If $p=2$ (linear eigenvalue problem), then these are all the eigenvalues of $\left(-\Delta, H_{0}^{1}(\Omega)\right)$.

Next we recall some basic definitions and facts from Morse theory and from [Cingolani and Vannella 2003; 2007], which we will need in order to produce a second nodal solution.

So, as before, let $X$ be a Banach space and $\left(Y_{1}, Y_{2}\right)$ a topological pair such that $Y_{2} \subseteq Y_{1} \subseteq X$. For every integer $k \geq 0$, by $H_{k}\left(Y_{1}, Y_{2}\right)$ we denote the $k$-th-relative singular homology group with integer coefficients for the pair $\left(Y_{1}, Y_{2}\right)$. For $k<0$, $H_{k}\left(Y_{1}, Y_{2}\right)=0$.

Given $\varphi \in C^{1}(X)$ and $c \in \mathbb{R}$, we introduce the sets

$$
\varphi^{c}=\{x \in X: \varphi(x) \leq c\}, \quad K_{\varphi}=\left\{x \in X: \varphi^{\prime}(x)=0\right\}, \quad K_{\varphi}^{c}=\left\{x \in K_{\varphi}: \varphi(x)=c\right\}
$$


The critical groups of $\varphi \in C^{1}(X)$ at an isolated critical point $x \in X$ with $\varphi(x)=c$ (i.e., $x \in K_{\varphi}^{c}$ ) are defined by

$$
C_{k}(\varphi, x)=H_{k}\left(\varphi^{c} \cap U, \varphi^{c} \cap U \backslash\{x\}\right) \text { for all } k \geq 0,
$$

where $U$ is a neighborhood of $x$ such that $K_{\varphi} \cap \varphi^{c} \cap U=\{x\}$. The excision property of singular homology theory implies that the above definition of critical groups is independent of the particular choice of the neighborhood $U$.

Now suppose that $\varphi \in C^{1}(X)$ satisfies the C-condition and $\inf \varphi\left(K_{\varphi}\right)>-\infty$. Let $c<\inf \varphi\left(K_{\varphi}\right)$. The critical groups of $\varphi$ at infinity are defined by

$$
C_{k}(\varphi, \infty)=H_{k}\left(X, \varphi^{c}\right) \text { for all } k \geq 0 .
$$

The second deformation theorem (see, for example, [Gasiński and Papageorgiou 2006, p. 628]), implies that this definition is independent of the level $c<\inf \varphi\left(K_{\varphi}\right)$.

Suppose that $K_{\varphi}$ is finite and define

$$
M(t, x)=\sum_{k \geq 0} \operatorname{rank} C_{k}(\varphi, x) t^{k} \quad \text { for all } t \in \mathbb{R}, \text { all } x \in K_{\varphi}
$$

and

$$
P(t, \infty)=\sum_{k \geq 0} \operatorname{rank} C_{k}(\varphi, \infty) t^{k} \quad \text { for all } t \in \mathbb{R} .
$$

The Morse relation says that

$$
\sum_{x \in K_{\varphi}} M(t, x)=P(t, \infty)+(1+t) Q(t)
$$

where $Q(t)=\sum_{k \geq 0} \beta_{k} t^{k}$ is a formal series in $t \in \mathbb{R}$ with integer coefficients $\beta_{k}$.

Let $H$ be a Hilbert space, $x$ a point in $H$, and $U$ a neighborhood of $x$. Let $\varphi \in C^{2}(U)$. If $x \in K_{\varphi}$, then the Morse index $\mu=\mu(x)$ of $x$ is defined to be the supremum of the dimensions of the vector subspaces of $H$ on which $\varphi^{\prime \prime}(x)$ is negative definite. The nullity $v(x)$ of $x \in K_{\varphi}$ is the dimension of $\operatorname{ker} \varphi^{\prime \prime}(x)$. We say that $x \in K_{\varphi}$ is nondegenerate if $\varphi^{\prime \prime}(x)$ is invertible (i.e., $\left.v(x)=0\right)$. If $\varphi \in C^{2}(U)$ and $x \in K_{\varphi}$ is nondegenerate with Morse index $\mu$, then

$$
C_{k}(\varphi, x)=\delta_{k, \mu} \mathbb{Z} \quad \text { for all } k \geq 0,
$$

where $\delta_{k, \mu}$ is the Kronecker symbol.

As mentioned in the introduction, to produce a second nodal solution, we will use some facts from [Cingolani and Vannella 2003; 2007]. Suppose $f: \Omega \times \mathbb{R} \rightarrow \mathbb{R}$ is a measurable function such that, for a.a. $z \in \Omega, f(z, \cdot) \in C^{1}(\mathbb{R})$ and

$$
\left|f_{x}^{\prime}(z, x)\right| \leq \tilde{\alpha}(z)+\tilde{c}|x|^{r-2} \quad \text { for a.a. } z \in \Omega \text {, all } x \in \mathbb{R},
$$


with $\tilde{\alpha} \in L^{\infty}(\Omega)_{+}, \tilde{c}>0$ and $p \leq r<p^{*}$. We set $F(z, x)=\int_{0}^{x} f(z, s) d s$ and consider the $C^{2}$-functional $\varphi: W_{0}^{1, p}(\Omega) \rightarrow \mathbb{R}$ defined by

$$
\varphi(u)=\frac{1}{p}\|D u\|_{p}^{p}+\frac{1}{2}\|D u\|_{2}^{2}-\int_{\Omega} F(z, u(z)) d z \quad \text { for all } u \in W_{0}^{1, p}(\Omega) .
$$

For all $u, v, y \in W_{0}^{1, p}(\Omega)$, we have (see [Cingolani and Vannella 2003])

$$
\begin{aligned}
\left\langle\varphi^{\prime \prime}(u) v, y\right\rangle & =\int_{\Omega}\left(1+\|D u\|^{p-2}\right)(D v, D y)_{\mathbb{R}^{N}} d z \\
& +(p-2) \int_{\Omega}\|D u\|^{p-4}(D u, D v)_{\mathbb{R}^{N}}(D u, D y)_{\mathbb{R}^{N}} d z-\int_{\Omega} f_{x}^{\prime}(z, u) v y d z .
\end{aligned}
$$

Here $\langle\cdot, \cdot\rangle$ denotes the duality brackets for the pair consisting of the spaces

$$
W^{-1, p^{\prime}}(\Omega)=W_{0}^{1, p}(\Omega)^{*} \quad \text { and } \quad W_{0}^{1, p}(\Omega), \quad \text { where } \frac{1}{p}+\frac{1}{p^{\prime}}=1 .
$$

Suppose that $u_{0} \in K_{\varphi}$. Nonlinear regularity theory (see [Ladyzhenskaya and Ural'tseva 1968; Lieberman 1991]) implies that $u_{0} \in C_{0}^{1}(\bar{\Omega})$. It follows that

$$
b(\cdot)=\left\|D u_{0}(\cdot)\right\|^{(p-4) / 2} D u_{0}(\cdot) \in L^{\infty}\left(\Omega, \mathbb{R}^{N}\right) .
$$

Let $H_{b}$ be the completion of $C_{c}^{\infty}(\Omega)$ under the inner product

$$
(v, y)_{b}=\int_{\Omega}\left[\left(1+\|b\|^{2}\right)(D v, D y)_{\mathbb{R}^{N}}+(p-2)(b, D v)_{\mathbb{R}^{N}}(b, D y)_{\mathbb{R}^{N}}\right] d z .
$$

Denote by $\|\cdot\|_{b}$ the corresponding norm. Clearly $\|\cdot\|_{b}$ is equivalent to the usual Sobolev norm of $H_{0}^{1}(\Omega)$, so $H_{b}$ and $H_{0}^{1}(\Omega)$ are isomorphic. Since $p>2, W_{0}^{1, p}(\Omega)$ is embedded continuously into $H_{b}$. Let $L_{b} \in \mathscr{L}\left(H_{b}, H_{b}^{*}\right)$ be defined by

$$
\left\langle L_{b}(v), y\right\rangle_{b}=(v, y)_{b}-\int_{\Omega} f_{x}^{\prime}\left(z, u_{0}\right) v y d z \quad \text { for all } v, y \in H_{b} .
$$

Then $L_{b}$ is a Fredholm operator of index zero and it is the extension of $\varphi^{\prime \prime}\left(u_{0}\right)$ on $H_{b}$. We consider the orthogonal direct sum decomposition

$$
H_{b}=H^{-} \oplus H^{0} \oplus H^{+},
$$

where $H^{-}, H^{0}, H^{+}$are the negative, null and positive spaces according to the spectral decomposition of $L_{b}$ in $L^{2}(\Omega)$. Then $H^{-}$and $H^{0}$ are finite-dimensional and, since $u_{0} \in C_{0}^{1}(\bar{\Omega})$, standard regularity theory implies that

$$
H^{-} \oplus H^{0} \subseteq W_{0}^{1, p}(\Omega) \cap L^{\infty}(\Omega) .
$$

We set $V=H^{-} \oplus H^{0}$ and $W=W_{0}^{1, p}(\Omega) \cap H^{+}$. Then $W_{0}^{1, p}(\Omega)=V \oplus W$ and, by [Cingolani and Vannella 2003, p. 279], there exists $c>0$ such that

$$
\left\langle\varphi^{\prime \prime}\left(u_{0}\right) v, v\right\rangle \geq c\|v\|_{b}^{2} \quad \text { for all } v \in W .
$$


In what follows, for every $r \in(1,+\infty)$, we denote by

$$
A_{r}: W_{0}^{1, r}(\Omega) \rightarrow W^{-1, r^{\prime}}(\Omega), \quad \frac{1}{r}+\frac{1}{r^{\prime}}=1,
$$

the nonlinear map defined by

$$
\left\langle A_{r}(u), y\right\rangle=\int_{\Omega}\|D u\|^{r-2}(D u, D y)_{\mathbb{R}^{N}} d z \quad \text { for all } u, y \in W_{0}^{1, r}(\Omega) .
$$

If $r=2$, then we set $A_{2}=A \in \mathscr{L}\left(H_{0}^{1}(\Omega), H^{-1}(\Omega)\right)$. The next result can be found in [Gasiński and Papageorgiou 2006, pp. 745-746].

Proposition 4. If $A_{r}: W_{0}^{1, r}(\Omega) \rightarrow W^{-1, r^{\prime}}(\Omega)$ is defined by (5), then $A_{r}$ is continuous, monotone (hence maximal monotone) and of type $(S)_{+}$; that is, if $u_{n}$ converges weakly to $u$ in $W_{0}^{1, p}(\Omega)$ and $\lim \sup _{n \rightarrow+\infty}\left\langle A_{r}\left(u_{n}\right), u_{n}-u\right\rangle \leq 0$, then $u_{n} \rightarrow u$ in $W_{0}^{1, r}(\Omega)$.

Throughout this paper by $\|\cdot\|$ we denote the norm of $W_{0}^{1, p}(\Omega)$. By virtue of Poincaré's inequality, $\|u\|=\|D u\|_{p}$ for all $u \in W_{0}^{1, p}(\Omega)$. By $\|\cdot\|$ we will also denote the norm of $\mathbb{R}^{N}$. No confusion is possible, since it will always be clear from the context which norm we mean.

For $x \in \mathbb{R}$, we define

$$
x^{ \pm}=\max \{ \pm x, 0\} .
$$

Then for $u \in W_{0}^{1, p}(\Omega)$ we set

$$
u^{ \pm}(\cdot)=u(\cdot)^{ \pm} .
$$

We know that $u^{ \pm} \in W_{0}^{1, p}(\Omega)$ and

$$
|u|=u^{+}+u^{-} \quad \text { and } \quad u=u^{+}-u^{-} .
$$

By $|\cdot|_{\mathbb{R}^{N}}$ we denote the Lebesgue measure on $\mathbb{R}^{N}$.

Finally, if $g: \Omega \times \mathbb{R} \rightarrow \mathbb{R}$ is a measurable function (for example, if $(z, x) \rightarrow g(z, x)$ is a Carathéodory function), then we set

$$
N_{g}(u)(\cdot)=g(\cdot, u(\cdot)) \quad \text { for all } u \in W_{0}^{1, p}(\Omega) .
$$

\section{Constant sign solutions}

In this section, we produce four nontrivial smooth solutions of constant sign, two positive and two negative. The hypotheses on the reaction $f(z, x)$ are the following:

Hypotheses $\mathbf{H}$. (i) $f: \Omega \times \mathbb{R} \rightarrow \mathbb{R}$ is a Carathéodory function.

(ii) $f(z, 0)=0$ a.e. in $\Omega$.

(iii) $|f(z, x)| \leq \alpha(z)+c|x|^{p-1}$ for a.a. $z \in \Omega$, all $x \in \mathbb{R}$, with $\alpha \in L^{\infty}(\Omega)_{+}, c>0$. 
(iv) If $F(z, x)=\int_{0}^{x} f(z, s) d s$, then

and, for some $\tau>2$,

$$
\lim _{x \rightarrow \pm \infty} \frac{p F(z, x)}{|x|^{p}}=\hat{\lambda}_{1}(p) \quad \text { uniformly for a.a. } z \in \Omega,
$$

$$
\limsup _{x \rightarrow \pm \infty} \frac{f(z, x) x-p F(z, x)}{|x|^{\tau}} \leq \hat{\beta}<0 \quad \text { uniformly for a.a. } z \in \Omega .
$$

(v) There exist functions $w_{ \pm} \in W^{1, p}(\Omega) \cap C(\bar{\Omega})$ such that

$$
\begin{gathered}
w_{-}(z) \leq c_{-}<0<c_{+} \leq w_{+}(z) \quad \text { for all } z \in \bar{\Omega}, \\
\underset{\Omega}{\operatorname{ess} \sup } f\left(\cdot, w_{+}(\cdot)\right) \leq 0 \leq \underset{\Omega}{\operatorname{essinf}} f\left(\cdot, w_{-}(\cdot)\right),
\end{gathered}
$$

and $A_{p}\left(w_{-}\right)+A\left(w_{-}\right) \leq 0 \leq A_{p}\left(w_{+}\right)+A\left(w_{+}\right)$in $W^{-1, p^{\prime}}(\Omega)=W_{0}^{1, p}(\Omega)^{*}$.

(vi) For every $\rho>0$, there exists $\xi_{\rho}>0$ such that for a.a. $z \in \Omega$, the function $x \rightarrow f(z, x)+\xi_{\rho}|x|^{p-2} x$ is nondecreasing on $[-\rho, \rho]$.

(vii) There exist integer $m \geq 2$ and functions $\eta, \hat{\eta} \in L^{\infty}(\Omega)_{+}$such that

$$
\begin{aligned}
& \hat{\lambda}_{m}(2) \leq \eta(z) \leq \hat{\eta}(z) \leq \hat{\lambda}_{m+1}(2) \quad \text { a.e. in } \Omega, \quad \hat{\lambda}_{m}(2) \neq \eta, \quad \hat{\lambda}_{m+1}(2) \neq \hat{\eta} \\
& \text { and }
\end{aligned}
$$$$
\eta(z) \leq \liminf _{x \rightarrow 0} \frac{f(z, x)}{x} \leq \limsup _{x \rightarrow 0} \frac{f(z, x)}{x} \leq \hat{\eta}(z) \quad \text { uniformly for a.a. } z \in \Omega .
$$

Remarks. Hypothesis H(iv) implies that, asymptotically at $\pm \infty$, we have resonance with respect to the principal eigenvalue $\hat{\lambda}_{1}(p)>0$ from the right. Hence the energy functional of the problem, as we will see, is indefinite. Hypothesis $\mathrm{H}(\mathrm{v})$ is satisfied if we can find $c_{-}<0<c_{+}$such that $f\left(z, c_{+}\right)=f\left(z, c_{-}\right)=0$ a.e. in $\Omega$.

Example. The following function satisfies the hypotheses $\mathrm{H}$ (for simplicity, we drop the $z$-dependence):

$$
f(x)=\left\{\begin{array}{cc}
\eta\left(x-|x|^{r-2} x\right) & \text { if }|x| \leq 1 \\
\hat{\lambda}_{1}(p)\left(|x|^{p-2} x-|x|^{\tau-2} x\right) & \text { if }|x|>1
\end{array}\right.
$$

with $\eta \in\left(\hat{\lambda}_{m}(2), \hat{\lambda}_{m+1}(2)\right), m \geq 2$ and $r>2,1<\tau<p$.

We introduce the following truncations of $f(z, \cdot)$ :

$$
\hat{f}_{+}(z, x)=\left\{\begin{array}{cl}
0 & \text { if } x<0 \\
f(z, x) & \text { if } 0 \leq x \leq w_{+}(z), \\
f\left(z, w_{+}(z)\right) & \text { if } w_{+}(z)<x
\end{array}\right.
$$


and

$$
\hat{f}_{-}(z, x)=\left\{\begin{array}{cl}
f\left(z, w_{-}(z)\right) & \text { if } x<w_{-}(z), \\
f(z, x) & \text { if } w_{-}(z) \leq x \leq 0, \\
0 & \text { if } 0<x
\end{array}\right.
$$

Both are Carathéodory functions. Let $\hat{F}_{ \pm}(z, x)=\int_{0}^{x} \hat{f}_{ \pm}(z, s) d s$ and consider the $C^{1}$-functionals $\hat{\varphi}_{ \pm}: W_{0}^{1, p}(Z) \rightarrow \mathbb{R}$ defined by

$$
\hat{\varphi}_{ \pm}(u)=\frac{1}{p}\|D u\|_{p}^{p}+\frac{1}{2}\|D u\|_{2}^{2}-\int_{\Omega} \hat{F}_{ \pm}(z, u(z)) d z \quad \text { for all } u \in W_{0}^{1, p}(\Omega) .
$$

Also, let $\varphi: W_{0}^{1, p}(\Omega) \rightarrow \mathbb{R}$ be the energy (Euler) functional for problem (1) defined by

$$
\varphi(u)=\frac{1}{p}\|D u\|_{p}^{p}+\frac{1}{2}\|D u\|_{2}^{2}-\int_{\Omega} F(z, u(z)) d z \quad \text { for all } u \in W_{0}^{1, p}(\Omega) .
$$

Clearly, $\varphi \in C^{1}\left(W_{0}^{1, p}(\Omega)\right)$.

First, we produce two nontrivial constant sign smooth solutions of (1).

Proposition 5. If hypotheses $\mathrm{H}(\mathrm{iii})$, (v), (vi), (vii) hold, then problem (1) has at least the two nontrivial constant sign smooth solutions

$$
u_{0} \in \operatorname{int} C_{+} \quad \text { and } \quad v_{0} \in-\operatorname{int} C_{+},
$$

and both are local minimizers of $\varphi$.

Proof. First we produce the positive solution.

From (6) we see that $\hat{\varphi}_{+}$is coercive. Also, using the Sobolev embedding theorem, we can check easily that $\hat{\varphi}_{+}$is sequentially weakly lower semicontinuous. So, by the Weierstrass theorem, we can find $u_{0} \in W_{0}^{1, p}(\Omega)$ such that

$$
\hat{\varphi}_{+}\left(u_{0}\right)=\inf \left[\hat{\varphi}_{+}(u): u \in W_{0}^{1, p}(\Omega)\right]=m_{+} .
$$

By virtue of hypothesis $\mathrm{H}\left(\right.$ vii), we can find $\vartheta>\hat{\lambda}_{1}(2)$ and $0<\delta<\min \left\{c_{+},-c_{-}\right\}$ such that

$$
F(z, x) \geq \frac{1}{2} \vartheta x^{2} \quad \text { for a.a. } z \in \Omega \text {, all }|x| \leq \delta .
$$

Let $t \in(0,1)$ be small such that $t \hat{u}_{1,2}(z) \in[0, \delta]$ for all $z \in \bar{\Omega}$ (recall that $\left.\hat{u}_{1,2} \in \operatorname{int} C_{+}\right)$. Then

$$
\begin{aligned}
\hat{\varphi}_{+}\left(t \hat{u}_{1,2}\right) & =\frac{t^{p}}{p}\left\|D \hat{u}_{1,2}\right\|_{p}^{p}+\frac{t^{2}}{2} \hat{\lambda}_{1}(2)-\int_{\Omega} \hat{F}_{+}\left(z, t \hat{u}_{1,2}\right) d z \\
& \leq \frac{t^{p}}{p}\left\|D \hat{u}_{1,2}\right\|_{p}^{p}+\frac{t^{2}}{2}\left[\hat{\lambda}_{1}(2)-\vartheta\right]
\end{aligned}
$$

(see (8) and recall that $\left\|\hat{u}_{1,2}\right\|_{2}=1$ ). 
Since $\vartheta>\hat{\lambda}_{1}(2)$ and $p>2$, by choosing $t \in(0,1)$ even smaller if necessary, we have $\hat{\varphi}_{+}\left(t \hat{u}_{1,2}\right)<0$, which implies $\hat{\varphi}_{+}\left(u_{0}\right)=\hat{m}_{+}<0=\hat{\varphi}_{+}(0)$ (see (7)); hence $u_{0} \neq 0$.

From (7) we have $\hat{\varphi}_{+}^{\prime}\left(u_{0}\right)=0$, which implies

$$
A_{p}\left(u_{0}\right)+A\left(u_{0}\right)=N_{\widehat{f}_{+}}\left(u_{0}\right) .
$$

On (9) we act with $-u_{0}^{-} \in W_{0}^{1, p}(\Omega)$ and obtain $u_{0} \geq 0, u_{0} \neq 0$ (see (6)). Also, we act with $\left(u_{0}-w_{+}\right)^{+} \in W_{0}^{1, p}(\Omega)$. Then

$$
\begin{aligned}
\left\langle A_{p}\left(u_{0}\right),\right. & \left.\left(u_{0}-w_{+}\right)^{+}\right\rangle+\left\langle A\left(u_{0}\right),\left(u_{0}-w_{+}\right)^{+}\right\rangle \\
& =\int_{\Omega} \hat{f}_{+}\left(z, u_{0}\right)\left(u_{0}-w_{+}\right)^{+} d z=\int_{\Omega} f\left(z, w_{+}\right)\left(u_{0}-w_{+}\right)^{+} d z \quad \text { (see (6)) } \\
\leq & \left\langle A_{p}\left(w_{+}\right)+A\left(w_{+}\right),\left(u_{0}-w_{+}\right)^{+}\right\rangle,
\end{aligned}
$$

by hypothesis $\mathrm{H}(\mathrm{v})$. Therefore

$$
\begin{aligned}
\int_{\left\{u_{0}>w_{+}\right\}}\left(\left\|D u_{0}\right\|^{p-2} D u_{0}-\left\|D w_{+}\right\|^{p-2} D w_{+}, D u_{0}-D w_{+}\right)_{\mathbb{R}^{N}} d z \\
+\left\|D\left(u_{0}-w_{+}\right)^{+}\right\|_{2}^{2} \leq 0 .
\end{aligned}
$$

It follows that $u_{0} \leq w_{+}$.

So, we have proved that

$$
u_{0} \in\left[0, w_{+}\right]=\left\{u \in W_{0}^{1, p}(\Omega): 0 \leq u(z) \leq w_{+}(z) \text { a.e. in } \Omega\right\} .
$$

Then (9) becomes $A_{p}\left(u_{0}\right)+A\left(u_{0}\right)=N_{f}\left(u_{0}\right)$ (see (6)), and hence

$$
-\Delta_{p} u_{0}(z)-\Delta u_{0}(z)=f\left(z, u_{0}(z)\right) \quad \text { a.e. in } \Omega,\left.\quad u_{0}\right|_{\partial \Omega}=0 .
$$

From (10) and [Ladyzhenskaya and Ural'tseva 1968, Theorem 7.1, p. 286], we have $u_{0} \in L^{\infty}(\Omega)$. We can apply the regularity result of [Lieberman 1991, p. 320] and have $u_{0} \in C_{+} \backslash\{0\}$. Note that

$$
A_{p}\left(u_{0}\right)+A\left(u_{0}\right)-N_{f}\left(u_{0}\right)=0 \leq A_{p}\left(w_{+}\right)+A\left(w_{+}\right)-N_{f}\left(w_{+}\right) \text {in } W^{-1, p^{\prime}}(\Omega),
$$

by $\mathrm{H}(\mathrm{v})$, and, for a.a. $z \in \Omega$ and all $x, y \in[-\rho, \rho]$ with $x>y$, we have, by $\mathrm{H}(\mathrm{vi})$,

$$
f(z, x)-f(z, y) \geq-\xi_{\rho}(x-y),
$$

Let $a(\xi)=\|\xi\|^{p-2} \xi+\xi$ for all $\xi \in \mathbb{R}^{N}$. Then $a \in C^{1}\left(\mathbb{R}^{N}\right)$,

$$
\nabla a(\xi)=\|\xi\|^{p-2}\left(I+(p-2) \frac{\xi \otimes \xi}{\|\xi\|^{2}}\right)+I
$$

and

$$
\operatorname{div} a(D u)=\Delta_{p} u+\Delta u \quad \text { for all } u \in W_{0}^{1, p}(\Omega) .
$$


We have $(\nabla a(\xi) y, y)_{\mathbb{R}^{N}} \geq\|y\|^{2}$ for all $\xi, y \in \mathbb{R}^{N}$ and so we can apply [Pucci and Serrin 2007, Theorem 2.5.3, p. 37] and infer, via H(v), that

$$
u_{0}(z)<w_{+}(z) \text { for all } z \in \bar{\Omega} \text {. }
$$

Let $\rho=\max \left\{\left\|w_{+}\right\|_{\infty},\left\|w_{-}\right\|_{\infty}\right\}$. By virtue of $\mathrm{H}(\mathrm{vi})$ and (10), we have

$$
-\Delta_{p} u_{0}(z)-\Delta u_{0}(z)+\hat{\xi}_{\rho} u_{0}(z)^{p-1}=f\left(z, u_{0}(z)\right)+\hat{\xi}_{\rho} u_{0}(z)^{p-1} \geq 0 \quad \text { a.e. in } \Omega \text {, }
$$

and hence

$$
\Delta_{p} u_{0}(z)+\Delta u_{0}(z) \leq \hat{\xi}_{\rho} u_{0}(z)^{p-1} \quad \text { a.e. in } \Omega .
$$

Invoking the boundary point theorem of Pucci and Serrin [2007, Theorem 5.5.1, p. 120] we have $u_{0} \in \operatorname{int} C_{+}$. Therefore

$$
u_{0} \in \operatorname{int}_{C_{0}^{1}(\bar{\Omega})}\left[0, w_{+}\right] .
$$

It is clear from (6) that $\left.\hat{\varphi}_{+}\right|_{\left[0, w_{+}\right]}=\left.\varphi\right|_{\left[0, w_{+}\right]}$. Therefore $u_{0}$ is a local $C_{0}^{1}(\bar{\Omega})$ minimizer of $\varphi$ and so by Theorem 2 it is also a local $W_{0}^{1, p}(\Omega)$-minimizer of $\varphi$.

Similarly, working this time with $\hat{\varphi}_{-}$, we produce another constant sign smooth solution $v_{0} \in-\operatorname{int} C_{+}$which is a local minimizer of $\varphi$.

Using $u_{0} \in \operatorname{int} C_{+}, v_{0} \in-\operatorname{int} C_{+}$, we can produce two more nontrivial constant sign smooth solutions.

Proposition 6. If hypotheses $\mathrm{H}$ hold and $K_{\varphi}$ is finite, problem (1) has at least four nontrivial constant sign smooth solutions

$$
\begin{aligned}
& u_{0}, \hat{u} \in \operatorname{int} C_{+} \quad \text { with } \hat{u}-u_{0} \in \operatorname{int} C_{+} \\
& v_{0}, \hat{v} \in-\operatorname{int} C_{+} \quad \text { with } v_{0}-\hat{v} \in \operatorname{int} C_{+} .
\end{aligned}
$$

Proof. From Proposition 5 we already have two solutions $u_{0} \in \operatorname{int} C_{+}$and $v_{0} \in$ - int $C_{+}$.

Next we produce the second nontrivial positive smooth solution. To this end, we introduce the following truncation of $f(z, \cdot)$ :

$$
h_{+}(z, x)=\left\{\begin{array}{cl}
f\left(z, u_{0}(z)\right) & \text { if } x \leq u_{0}(z), \\
f(z, x) & \text { if } u_{0}(z)<x .
\end{array}\right.
$$

This is a Carathéodory function. We set $H_{+}(z, x)=\int_{0}^{x} h_{+}(z, s) d s$ and consider the $C^{1}$-functional $\psi_{+}: W_{0}^{1, p}(\Omega) \rightarrow \mathbb{R}$ defined by

$$
\psi_{+}(u)=\frac{1}{p}\|D u\|_{p}^{p}+\frac{1}{2}\|D u\|_{2}^{2}-\int_{\Omega} H_{+}(z, u(z)) d z \quad \text { for all } u \in W_{0}^{1, p}(\Omega) .
$$

Claim 1. The functional $\psi_{+}$satisfies the C-condition. 
Proof. Let $\left\{u_{n}\right\}_{n \geq 1} \subseteq W_{0}^{1, p}(\Omega)$ be a sequence such that

$$
\left|\psi_{+}\left(u_{n}\right)\right| \leq M_{1} \quad \text { for some } M_{1}>0, \text { all } n \geq 1
$$

and

$$
\left(1+\left\|u_{n}\right\|\right) \psi_{+}^{\prime}\left(u_{n}\right) \rightarrow 0 \quad \text { in } W^{-1, p^{\prime}}(\Omega) \text { as } n \rightarrow \infty .
$$

From (13) we have

$$
\left|\left\langle A_{p}\left(u_{n}\right), g\right\rangle+\left\langle A\left(u_{n}\right), g\right\rangle-\int_{\Omega} h_{+}\left(z, u_{n}\right) g d z\right| \leq \frac{\varepsilon_{n}\|g\|}{1+\left\|u_{n}\right\|}
$$

for all $g \in W_{0}^{1, p}(\Omega)$, with $\varepsilon_{n} \downarrow 0$.

In (14) we choose $g=-u_{n}^{-} \in W_{0}^{1, p}(\Omega)$. Then we get

$$
\left\|D u_{n}^{-}\right\|_{p}^{p}+\left\|D u_{n}^{-}\right\|_{2}^{2}-\int_{\Omega} f\left(z, u_{0}\right)\left(-u_{n}^{-}\right) d z \leq \varepsilon_{n} \quad \text { for all } n \geq 1,
$$

by (11); this implies that $\left\|D u_{n}^{-}\right\|_{p}^{p} \leq c_{1}\left\|u_{n}^{-}\right\|$for some $c_{1}>0$ and all $n \geq 1$ (by H(iii)), and we conclude, since $p>1$, that

$$
\left\{u_{n}^{-}\right\}_{n \geq 1} \subseteq W_{0}^{1, p}(\Omega) \quad \text { is bounded. }
$$

We will show that $\left\{u_{n}\right\}_{n \geq 1} \subseteq W_{0}^{1, p}(\Omega)$ is bounded. Arguing by contradiction, because of (15) and by passing to a suitable subsequence if necessary, we may assume that $\left\|u_{n}^{+}\right\| \rightarrow \infty$. We set $y_{n}=u_{n}^{+} /\left\|u_{n}^{+}\right\|, n \geq 1$. Then $\left\|y_{n}\right\|=1$ for all $n \geq 1$ and so we may assume that

$$
y_{n} \stackrel{w}{\longrightarrow} y \text { in } W_{0}^{1, p}(\Omega) \text { and } y_{n} \rightarrow y \text { in } L^{p}(\Omega) \quad \text { as } n \rightarrow \infty,
$$

where $\stackrel{w}{\longrightarrow}$ indicates weak convergence. From (14), we have

$$
\left|\left\langle A_{p}\left(y_{n}\right), g\right\rangle+\frac{1}{\left\|u_{n}^{+}\right\|^{p-2}}\left\langle A\left(y_{n}\right), g\right\rangle-\int_{\Omega} \frac{h_{+}\left(z, u_{n}^{+}\right)}{\left\|u_{n}^{+}\right\|^{p-1}} g d z\right| \leq \varepsilon_{n}^{\prime}\|g\|,
$$

with $\varepsilon_{n}^{\prime} \rightarrow 0$ (see (15)).

Hypothesis $\mathrm{H}$ (iii) and (11) imply that

$$
\left\{\frac{N_{h_{+}}\left(u_{n}^{+}\right)}{\left\|u_{n}^{+}\right\|^{p-1}}\right\}_{n \geq 1} \subseteq L^{p^{\prime}}(\Omega) \quad \text { is bounded. }
$$

From (18) and using hypothesis H(iv), as in the proof of Proposition 30 of [Aizicovici et al. 2008], we have

$$
\frac{N_{h_{+}}\left(u_{n}^{+}\right)}{\left\|u_{n}^{+}\right\|^{p-1}} \stackrel{w}{\longrightarrow} \beta=\hat{\lambda}_{1}(p) y^{p-1} \quad \text { in } L^{p^{\prime}}(\Omega) .
$$


Also, if in (17) we choose $g=y_{n}-y \in W_{0}^{1, p}(\Omega)$, pass to the limit as $n \rightarrow \infty$ and use (16) and (19), we obtain

$$
\lim _{n \rightarrow \infty}\left[\left\langle A_{p}\left(y_{n}\right), y_{n}-y\right\rangle+\left\langle A\left(y_{n}\right), y_{n}-y\right\rangle\right]=0,
$$

from which we get successively

$\limsup \left[\left\langle A_{p}\left(y_{n}\right), y_{n}-y\right\rangle+\left\langle A(y), y_{n}-y\right\rangle\right] \leq 0 \quad$ (since $A$ is monotone), $n \rightarrow \infty$

$$
\begin{aligned}
\limsup _{n \rightarrow \infty}\left\langle A_{p}\left(y_{n}\right), y_{n}-y\right\rangle \leq 0 & (\text { see }(16)), \\
y_{n} \rightarrow y \quad \text { in } W_{0}^{1, p}(\Omega) & (\text { see Proposition 4). }
\end{aligned}
$$

The upshot is that

$$
\|y\|=1, \quad y \geq 0 \text {. }
$$

Passing to the limit as $n \rightarrow \infty$ in (17) and using (19) and (20), we see that

$$
\left\langle A_{p}(y), g\right\rangle=\hat{\lambda}_{1}(p) \int_{\Omega} y^{p-1} g d z \quad \text { for all } g \in W_{0}^{1, p}(\Omega),
$$

since $p>2$ and $\left\|u_{n}^{+}\right\| \rightarrow \infty$. This yields $A_{p}(y) \hat{\lambda}_{1}(p) y^{p-1}$ and so

$$
-\Delta_{p} y(z)=\hat{\lambda}_{1}(p) y(z)^{p-1} \quad \text { a.e. in } \Omega,\left.\quad y\right|_{\partial \Omega}=0,
$$

implying, in view of (20), that

$$
y=\lambda \hat{u}_{1, p} \quad \text { for some } \lambda>0 .
$$

Therefore $y(z)>0$ for all $z \in \Omega$ and this implies that $u_{n}^{+}(z) \rightarrow+\infty$ for all $z \in \Omega$. Then, by virtue of hypothesis H(iv), we have

$$
\limsup _{n \rightarrow \infty} \frac{f\left(z, u_{n}^{+}(z)\right) u_{n}^{+}(z)-p F\left(z, u_{n}^{+}(z)\right)}{\left|u_{n}^{+}(z)\right|^{\tau}} \leq \hat{\beta}<0 \quad \text { for a.a. } z \in \Omega,
$$

or again, in view of (11),

$$
\limsup _{n \rightarrow \infty} \frac{h_{+}\left(z, u_{n}^{+}(z)\right) u_{n}^{+}(z)-p H_{+}\left(z, u_{n}^{+}(z)\right)}{\left|u_{n}^{+}(z)\right|^{\tau}} \leq \hat{\beta}<0 \quad \text { for a.a. } z \in \Omega .
$$

Hypothesis H(iv) and Fatou's lemma, together with (21) and (22), imply that

$$
\limsup _{n \rightarrow \infty} \frac{1}{\left\|u_{n}^{+}\right\|^{\tau}} \int_{\Omega}\left[h_{+}\left(z, u_{n}^{+}\right) u_{n}^{+}(z)-p H_{+}\left(z, u_{n}^{+}\right)\right] d z<0
$$

On the other hand, from (12) and (15), we have

$$
-M_{2} \leq\left\|D u_{n}^{+}\right\|_{p}^{p}+\frac{p}{2}\left\|D u_{n}^{+}\right\|_{2}^{2}-\int_{\Omega} p H_{+}\left(z, u_{n}^{+}\right) d z
$$


for some $M_{2}>0$ and all $n \geq 1$.

Also, if we choose $g=u_{n}^{+} \in W_{0}^{1, p}(\Omega)$ in (14), then

$$
-\varepsilon_{n} \leq-\left\|D u_{n}^{+}\right\|_{p}^{p}-\left\|D u_{n}^{+}\right\|_{2}^{2}+\int_{\Omega} h_{+}\left(z, u_{n}^{+}\right) u_{n}^{+} d z \quad \text { for all } n \geq 1 .
$$

Adding (24) and (25), we obtain

$$
-M_{3} \leq \int_{\Omega}\left[h_{+}\left(z, u_{n}^{+}\right) u_{n}^{+}-p H_{+}\left(z, u_{n}^{+}\right)\right] d z+\left(\frac{p}{2}-1\right)\left\|D u_{n}^{+}\right\|_{2}^{2}
$$

for some $M_{3}>0$ and all $n \geq 1$, whence (since $p>2$ )

$$
-\frac{M_{3}}{\left\|u_{n}^{+}\right\|^{\tau}} \leq \frac{1}{\left\|u_{n}^{+}\right\|^{\tau}} \int_{\Omega}\left[h_{+}\left(z, u_{n}^{+}\right) u_{n}^{+}-p H_{+}\left(z, u_{n}^{+}\right)\right] d z+c_{2}\left(\frac{p}{2}-1\right) \frac{1}{\left\|u_{n}^{+}\right\|^{\tau-2}}
$$

for some $c_{2}>0$ and all $n \geq 1$, and finally, since $\tau>2$ and $p>2$,

$$
0 \leq \liminf _{n \rightarrow \infty} \frac{1}{\left\|u_{n}^{+}\right\|^{\tau}} \int_{\Omega}\left[h_{+}\left(z, u_{n}^{+}\right) u_{n}^{+}-p H_{+}\left(z, u_{n}^{+}\right)\right] d z .
$$

Comparing (23) and (26), we reach a contradiction.

This proves that $\left\{u_{n}^{+}\right\}_{n \geq 1} \subseteq W_{0}^{1, p}(\Omega)$ is bounded; hence $\left\{u_{n}\right\}_{n \geq 1} \subseteq W_{0}^{1, p}(\Omega)$ is bounded, by (15). So, we may assume that

$$
u_{n} \stackrel{w}{\longrightarrow} u \text { in } W_{0}^{1, p}(\Omega) \quad \text { and } \quad u_{n} \rightarrow u \text { in } L^{p}(\Omega) .
$$

If in (14) we choose $g=u_{n}-u \in W_{0}^{1, p}(\Omega)$, pass to the limit as $n \rightarrow \infty$ and use (27), then, as before, exploiting the monotonicity of $A$, we have

$$
\limsup _{n \rightarrow \infty}\left\langle A_{p}\left(u_{n}\right), u_{n}-u\right\rangle \leq 0
$$

implying that $u_{n} \rightarrow u$ in $W_{0}^{1, p}(\Omega)$, by Proposition 4 . Hence $\psi_{+}$satisfies the Ccondition, and this proves Claim 1.

Claim 2. The function $u_{0}$ is a local minimizer of $\psi_{+}$.

Proof. We may assume that $K_{\varphi} \cap\left[0, w_{+}\right]=\left\{0, u_{0}\right\}$. Otherwise, let $y$ be a nontrivial element of $K_{\varphi} \cap\left[0, w_{+}\right]$distinct from $u_{0}$; as a nontrivial solution of (1), $y$ can be taken such that $u_{0} \leq y$, because (1) has a biggest solution in $\left[0, w_{+}\right]$(this is shown like Proposition 4.4 in [Filippakis et al. 2009]). Therefore, we are done if such a $y$ exists.

We introduce the following truncation of $h_{+}(z, \cdot)$ :

$$
\hat{h}_{+}(z, x)=\left\{\begin{array}{cl}
h_{+}(z, x) & \text { if } x \leq w_{+}(z), \\
h_{+}\left(z, w_{+}(z)\right) & \text { if } w_{+}(z)<x .
\end{array}\right.
$$

This is a Carathéodory function. We set $\hat{H}_{+}(z, x)=\int_{0}^{x} \hat{h}_{+}(z, s) d s$ and consider 
the $C^{1}$-functional $\hat{\psi}_{+}: W_{0}^{1, p}(\Omega) \rightarrow \mathbb{R}$ defined by

$$
\hat{\psi}_{+}(u)=\frac{1}{p}\|D u\|_{p}^{p}+\frac{1}{2}\|D u\|_{2}^{2}-\int_{\Omega} \hat{H}_{+}(z, u(z)) d z \quad \text { for all } u \in W_{0}^{1, p}(\Omega) .
$$

From (28) it is clear that $\hat{\psi}_{+}$is coercive. Also, it is sequentially weakly lower semicontinuous. Hence, we can find $\bar{u}_{0} \in W_{0}^{1, p}(\Omega)$ such that

$$
\hat{\psi}_{+}\left(\bar{u}_{0}\right)=\inf \left[\hat{\psi}_{+}(u): u \in W_{0}^{1, p}(\Omega)\right],
$$

which is to say $\hat{\psi}_{+}^{\prime}\left(\bar{u}_{0}\right)=0$; therefore

$$
A_{p}\left(\bar{u}_{0}\right)+A\left(\bar{u}_{0}\right)=N_{\widehat{h}_{+}}\left(\bar{u}_{0}\right) .
$$

On (29) first we act with $\left(u_{0}-\bar{u}_{0}\right)^{+} \in W_{0}^{1, p}(\Omega)$ and then with $\left(\bar{u}_{0}-w_{+}\right)^{+} \in$ $W_{0}^{1, p}(\Omega)$. Using (11), (28) and hypothesis $\mathrm{H}(\mathrm{v})$, this leads to

$$
\bar{u}_{0} \in\left[u_{0}, w_{+}\right]=\left\{u \in W_{0}^{1, p}(\Omega): u_{0}(z) \leq u(z) \leq w_{+}(z) \text { a.e. in } \Omega\right\} .
$$

Then (29) becomes $A_{p}\left(\bar{u}_{0}\right)+A\left(\bar{u}_{0}\right)=N_{f}\left(\bar{u}_{0}\right)$ by (11) and (28); thus $\bar{u}_{0} \in K_{\varphi} \cap$ $\left[0, w_{+}\right]$, which is to say $\bar{u}_{0}=u_{0}$.

From Proposition 5 and its proof, we have

$$
u_{0} \in \operatorname{int} C_{+} \quad \text { and } \quad u_{0}(z)<w_{+}(z) \quad \text { for all } z \in \bar{\Omega} .
$$

From (28) we infer that $\left.\psi_{+}\right|_{\left[0, w_{+}\right]}=\left.\hat{\psi}_{+}\right|_{\left[0, w_{+}\right]}$, so $u_{0}$ is a local $C_{0}^{1}(\bar{\Omega})$-minimizer of $\psi_{+}$. Applying Theorem 2, we see that $u_{0}$ is also a local $W_{0}^{1, p}(\Omega)$-minimizer of $\psi_{+}$, as we wished to show.

If $u \in K_{\psi_{+}}$, then

$$
A_{p}(u)+A(u)=N_{h_{+}}(u) .
$$

Acting with $\left(u_{0}-u\right)^{+} \in W_{0}^{1, p}(\Omega)$ and using (11), we show that $u_{0} \leq u$. Therefore

$$
K_{\psi_{+}} \subseteq\left[u_{0}\right)=\left\{u \in W_{0}^{1, p}(\Omega): u_{0}(z) \leq u(z) \text { for a.a. } z \in \Omega\right\} .
$$

By virtue of Claim 2, $u_{0} \in K_{\psi_{+}}$. Note that from (11) and (30) it follows that $K_{\psi_{+}} \subseteq K_{\varphi}$ and recall that by hypothesis $K_{\varphi}$ is finite. So, as in [Aizicovici et al. 2008, proof of Proposition 29], we can find $\rho \in(0,1)$ small such that

$$
\psi_{+}\left(u_{0}\right)<\inf \left[\psi_{+}(u):\left\|u-u_{0}\right\|=\rho\right]=\eta_{\rho}^{+} .
$$

Claim 3.

$$
\psi_{+}\left(t \hat{u}_{1, p}\right) \rightarrow-\infty \quad \text { as } t \rightarrow+\infty \text {. }
$$

Proof. By virtue of hypothesis $\mathrm{H}(\mathrm{iv})$, we can find $\hat{\beta}_{1} \in(\hat{\beta}, 0)$ and $M_{4}>\left\|u_{0}\right\|_{\infty}$ such that $f(z, x) x-p F(z, x) \leq \hat{\beta}_{1} x^{\tau}$ for a.a. $z \in \Omega$, all $x \geq M_{4}$. Thus

$$
h_{+}(z, x) x-p H_{+}(z, x) \leq \hat{\beta}_{1} x^{\tau}+c_{3}
$$


for a.a. $z \in \Omega$, all $x \geq M_{4}$, some $c_{3}>0$.

(Note that $F(z, x)=H_{+}(z, x)-H_{+}\left(z, u_{0}(z)\right)+f\left(z, u_{0}(z)\right) u_{0}(z)$ for a.a. $z \in \Omega$, all $x \geq M_{4}$.)

Without loss of generality, we may assume that $\tau<p$ (see H(iv)). We have

$$
\begin{aligned}
\frac{\mathrm{d}}{\mathrm{d} x}\left(\frac{H_{+}(z, x)}{x^{p}}\right) & =\frac{h_{+}(z, x) x^{p}-p x^{p-1} H_{+}(z, x)}{x^{2 p}} \\
& =\frac{h_{+}(z, x) x-p H_{+}(z, x)}{x^{p+1}} \leq \frac{\hat{\beta}_{1} x^{\tau}+c_{3}}{x^{p+1}} \quad \text { (see (32)) } \\
& =\hat{\beta}_{1} x^{\tau-p-1}+\frac{c_{3}}{x^{p+1}} .
\end{aligned}
$$

It follows that

$$
\frac{H_{+}(z, x)}{x^{p}}-\frac{H_{+}(z, y)}{y^{p}} \leq-\frac{\hat{\beta}_{1}}{p-\tau}\left(\frac{1}{x^{p-\tau}}-\frac{1}{y^{p-\tau}}\right)-\frac{c_{3}}{p}\left(\frac{1}{x^{p}}-\frac{1}{y^{p}}\right)
$$

for a.a. $z \in \Omega$, all $x \geq y \geq M_{4}$.

Letting $x \rightarrow+\infty$, using hypothesis $\mathrm{H}(\mathrm{iv})$ and recalling that $\tau<p$, we obtain

$$
\frac{\hat{\lambda}_{1}(p)}{p}-\frac{H_{+}(z, y)}{y^{p}} \leq \frac{\hat{\beta}_{1}}{p-\tau} \frac{1}{y^{p-\tau}}+\frac{c_{3}}{p} \frac{1}{y^{p}} \quad \text { for a.a. } z \in \Omega, \text { all } y \geq M_{4},
$$

or, upon multiplication by $y^{p}$ and with $c_{4}=c_{3} / p$,

$$
\frac{\hat{\lambda}_{1}(p)}{p} y^{p}-H_{+}(z, y) \leq \frac{\hat{\beta}_{1}}{p-\tau} y^{\tau}+c_{4} \quad \text { for a.a. } z \in \Omega \text {, all } y \geq 0 .
$$

Then, for $t>0$, we have

$$
\begin{aligned}
\psi_{+}\left(t \hat{u}_{1, p}\right) & =\frac{t^{p}}{p} \hat{\lambda}_{1}(p)\left\|\hat{u}_{1, p}\right\|_{p}^{p}+\frac{t^{2}}{2}\left\|D \hat{u}_{1, p}\right\|_{2}^{2}-\int_{\Omega} H_{+}\left(z, t \hat{u}_{1, p}\right) d z \\
& \leq \frac{\hat{\beta}_{1}}{p-\tau} t^{\tau}\left\|\hat{u}_{1, p}\right\|_{\tau}^{\tau}+\frac{t^{2}}{2}\left\|D \hat{u}_{1, p}\right\|_{2}^{2}+c_{4}|\Omega|_{N} \quad \text { (see (33)). }
\end{aligned}
$$

Since $\tau>2$ (see H(iv)) and $\hat{\beta}_{1}<0$, it follows from (34) that $\psi_{+}\left(t \hat{u}_{1, p}\right) \rightarrow-\infty$ as $t \rightarrow+\infty$. This proves Claim 3 .

Claims 1, 3 and (31) permit the use of Theorem 1, the mountain pass theorem. So, we can find $\hat{u} \in W_{0}^{1, p}(\Omega)$ such that

$$
\psi_{+}\left(u_{0}\right)<\eta_{\rho}^{+} \leq \psi_{+}(\hat{u})
$$

(see (31)) and

$$
\psi_{+}^{\prime}(\hat{u})=0 .
$$

From (35) we see that $\hat{u} \neq u_{0}$, while from (36) we have $\hat{u} \in\left[u_{0}\right)$ (see (30)). 
Therefore $\hat{u}$ is the second nontrivial positive solution of (1) (see (11)). Moreover, nonlinear regularity theory (see [Lieberman 1991]) implies that $\hat{u} \in \operatorname{int} C_{+}, u_{0} \leq \hat{u}$, $u_{0} \neq \hat{u}$. From the tangency principle of [Pucci and Serrin 2007, p. 35], we have

$$
u_{0}(z)<\hat{u}(z) \text { for all } z \in \Omega \text {. }
$$

Let $\rho=\|\hat{u}\|_{\infty}$ and let $\hat{\xi}>\hat{\xi}_{\rho}\left(\hat{\xi}_{\rho}>0\right.$ as postulated by hypothesis $\left.\mathrm{H}(\mathrm{vi})\right)$. We set

$$
h(z)=f\left(z, u_{0}(z)\right)+\hat{\xi} u_{0}(z)^{p-1} \quad \text { and } \quad \hat{h}(z)=f(z, \hat{u}(z))+\hat{\xi} \hat{u}(z)^{p-1} .
$$

Clearly, $h, \hat{h} \in L^{\infty}(\Omega)_{+}, h \prec \hat{h}$ (see H(vi) and recall $u_{0}(z)<\hat{u}(z)$ for all $z \in \Omega$ ). Moreover, $\hat{u} \in \operatorname{int} C_{+}$and so we can use Proposition 3 and infer that $\hat{u}-u_{0} \in \operatorname{int} C_{+}$.

Similarly, consider the truncation

$$
h_{-}(z, x)=\left\{\begin{array}{cl}
f(z, x) & \text { if } x<v_{0}(z), \\
f\left(z, v_{0}(z)\right) & \text { if } v_{0}(z) \leq x .
\end{array}\right.
$$

Arguing as before, we produce a second nontrivial negative solution $\hat{v} \in-\operatorname{int} C_{+}$ such that $v_{0}-\hat{v} \in \operatorname{int} C_{+}$.

\section{Nodal solutions}

In this section we produce nodal solutions for problem (1). Under the current hypotheses $\mathrm{H}$, we will produce a nodal solution, and subsequently, by strengthening the regularity on $f(z, \cdot)$ (see hypotheses $\widehat{\mathrm{H}}$ below), we will generate a second nodal solution. In this section, Morse theory is a basic tool.

Our strategy is the following. First we will show that problem (1) has extremal constant sign solutions; i.e., there is a smallest nontrivial positive solution $u_{+}$ of (1) and a biggest nontrivial negative solution $v_{-}$of $(1)$. By truncating $f(z, \cdot)$ at $\left\{v_{-}(z), u_{+}(z)\right\}$ and using variational methods and Morse theoretic techniques, we show that problem (1) has nontrivial solutions in the order interval $\left[v_{-}, u_{+}\right]$distinct from $v_{-}$and $u_{+}$. The extremality of $v_{-}$and $u_{+}$implies that such solutions are necessarily nodal. The nonhomogeneity of the differential operator $u \rightarrow-\Delta_{p} u-\Delta u$ creates difficulties, which we have to overcome. To this end, note that hypotheses $\mathrm{H}(\mathrm{iii})$, (vii) imply that we can find $c_{5}>\hat{\lambda}_{1}(2)$ and $c_{6}>0$ such that

$$
f(z, x) x \geq c_{5} x^{2}-c_{6}|x|^{p} \quad \text { for a.a. } z \in \Omega \text {, all } x \in \mathbb{R} .
$$

This growth estimate leads to the following Dirichlet problem

$$
-\Delta_{p} u(z)-\Delta u(z)=c_{5} u(z)-c_{6}|u(z)|^{p-2} u(z) \quad \text { in } \Omega,\left.\quad u\right|_{\partial \Omega}=0 .
$$

Proposition 7. Problem (37) has a unique nontrivial positive solution $u_{*} \in \operatorname{int} C_{+}$ and, since (37) is odd, $v_{*}=-u_{*} \in-\operatorname{int} C_{+}$is the unique nontrivial negative solution of (37). 
Proof. We consider the $C^{1}$-functional $\gamma_{+}: W_{0}^{1, p}(\Omega) \rightarrow \mathbb{R}$ defined by $\gamma_{+}(u)=\frac{1}{p}\|D u\|_{p}^{p}+\frac{1}{2}\|D u\|_{2}^{2}-\frac{c_{5}}{2}\left\|u^{+}\right\|_{2}^{2}+\frac{c_{5}}{p}\left\|u^{+}\right\|_{p}^{p} \quad$ for all $u \in W_{0}^{1, p}(\Omega)$.

Since $p>2$, it is clear that $\gamma_{+}$is coercive. Also, $\gamma_{+}$is sequentially weakly lower semicontinuous. Therefore, we can find $u_{*} \in W_{0}^{1, p}(\Omega)$ such that

$$
\gamma_{+}\left(u_{*}\right)=\inf \left[\gamma_{+}(u): u \in W_{0}^{1, p}(\Omega)\right]=m_{*}^{+} .
$$

Since $c_{5}>\hat{\lambda}_{1}(2)$ and $p>2$, for $t \in(0,1)$ small, we have $\gamma_{+}\left(t \hat{u}_{1,2}\right)<0$, which implies $\gamma_{+}\left(u_{*}\right)=m_{*}^{+}<0=\gamma_{+}(0)$ by (38); hence $u_{*} \neq 0$.

From (38) we have

$$
\gamma_{+}^{\prime}\left(u_{*}\right)=0
$$

and therefore

$$
A_{p}\left(u_{*}\right)+A\left(u_{*}\right)=c_{5} u_{*}^{+}-c_{6}\left(u_{*}^{+}\right)^{p-1} .
$$

On (39) we act with $-u_{*}^{-} \in W_{0}^{1, p}(\Omega)$ and infer that $u_{*} \geq 0, u_{*} \neq 0$. Hence $A_{p}\left(u_{*}\right)+A\left(u_{*}\right)=c_{5} u_{*}-c_{6} u_{*}^{p-1}$, and so

$$
-\Delta_{p} u_{*}(z)-\Delta u_{*}(z)=c_{5} u_{*}(z)-c_{6} u_{*}(z)^{p-1} \quad \text { a.e. in } \Omega,\left.\quad u_{*}\right|_{\partial \Omega}=0 .
$$

Nonlinear regularity theory (see [Ladyzhenskaya and Ural'tseva 1968; Lieberman 1991]) implies that $u_{*} \in C_{+} \backslash\{0\}$. Moreover, from the strong maximum principle of [Pucci and Serrin 2007, p. 34], we have $u_{*}(z)>0$ for all $z \in \Omega$. Then

$$
\Delta_{p} u_{*}(z)+\Delta u_{*}(z) \leq c_{6} u_{*}(z)^{p-1} \quad \text { a.e. in } \Omega,
$$

which in view of [Pucci and Serrin 2007, p. 120] leads to

$$
u_{*} \in \operatorname{int} C_{+} .
$$

This establishes the existence of a nontrivial positive smooth solution of (37).

Next we show the uniqueness of $u_{*} \in \operatorname{int} C_{+}$. To this end, we consider the integral functional $\beta_{+}: L^{1}(\Omega) \rightarrow \overline{\mathbb{R}}=\mathbb{R} \cup\{+\infty\}$ defined by

$$
\beta_{+}(u)=\left\{\begin{array}{cl}
\frac{1}{p}\left\|D u^{1 / 2}\right\|_{p}^{p}+\frac{1}{2}\left\|D u^{1 / 2}\right\|_{2}^{2} & \text { if } u \geq 0, u^{1 / 2} \in W_{0}^{1, p}(\Omega), \\
+\infty & \text { otherwise }
\end{array}\right.
$$

Let $G_{0}(t)=t^{p} / p+t^{2} / 2$ for all $t \geq 0$. Clearly $G_{0}$ is strictly convex and strictly increasing. We set $G(y)=G_{0}(\|y\|)$ for all $y \in \mathbb{R}^{N}$. From (40) we have

$$
\beta_{+}(u)=\left\{\begin{array}{cl}
\int_{\Omega} G\left(D u^{1 / 2}\right) d z & \text { if } u \geq 0, u^{1 / 2} \in W_{0}^{1, p}(\Omega), \\
+\infty & \text { otherwise }
\end{array}\right.
$$


Let $u_{1}, u_{2} \in \operatorname{dom} \beta_{+}$and set $y_{1}=u_{1}^{1 / 2}, y_{2}=u_{2}^{1 / 2}$. Then $y_{1}, y_{2} \in W_{0}^{1, p}(\Omega)$. We define

$$
y_{3}=\left(t u_{1}+(1-t) u_{2}\right)^{1 / 2} \in W_{0}^{1, p}(\Omega) \quad \text { with } t \in[0,1] .
$$

Then Lemma 4 of [Benguria et al. 1981] (see also [Díaz and Saá 1987, Lemma 1]) implies that

$$
\left\|D y_{3}(z)\right\| \leq\left(t\left\|D y_{1}(z)\right\|^{2}+(1-t)\left\|D y_{2}(z)\right\|^{2}\right)^{1 / 2} \text { a.e. in } \Omega,
$$

or again, since $G_{0}$ is increasing,

$$
G_{0}\left(\left\|D y_{3}(z)\right\|\right) \leq G_{0}\left(\left(t\left\|D y_{1}(z)\right\|^{2}+(1-t)\left\|D y_{2}(z)\right\|^{2}\right)^{1 / 2}\right) \quad \text { a.e. in } \Omega .
$$

The right-hand side is bounded above by $t G_{0}\left(\left\|D y_{1}(z)\right\|\right)+(1-t) G_{0}\left(\left\|D y_{2}(z)\right\|\right)$, since $t \rightarrow G_{0}\left(t^{1 / 2}\right)$ is convex. So from (42) we obtain successively

$$
\begin{array}{rlrl}
G\left(D y_{3}(z)\right) & \leq t G\left(D y_{1}(z)\right)+(1-t) G\left(D y_{2}(z)\right) & & \text { a.e. in } \Omega, \\
G\left(D\left(t u_{1}+(1-t) u_{2}\right)^{1 / 2}(z)\right) & \leq t G\left(D u_{1}^{1 / 2}(z)\right)+(1-t) G\left(D u_{2}^{1 / 2}(z)\right) & \text { a.e. in } \Omega,
\end{array}
$$

and finally, using (41), the convexity of $\beta_{+}$.

Let $u \in W_{0}^{1, p}(\Omega)$ be a nontrivial positive solution of the auxiliary problem (37). From the first part of the proof we have $u \in \operatorname{int} C_{+}$. Therefore $u^{2} \in \operatorname{dom} \beta_{+}$. Also, if $h \in C_{0}^{1}(\bar{\Omega})$ and $t \in(-1,1)$ is small, then $u^{2}+t h \in \operatorname{dom} \beta_{+}$. So, the Gâteaux derivative of $\beta_{+}$at $u^{2}$ in the direction $h$ exists. The chain rule and the density of $C_{0}^{1}(\bar{\Omega})$ in $W_{0}^{1, p}(\Omega)$ imply

$$
\beta_{+}^{\prime}\left(u^{2}\right)(h)=\int_{\Omega} \frac{-\Delta_{p} u-\Delta u}{u} h d z \quad \text { for all } h \in W_{0}^{1, p}(\Omega) .
$$

Similarly, if $v \in W_{0}^{1, p}(\Omega)$ is another nontrivial positive solution of (37), then $v \in \operatorname{int} C_{+}$and we have

$$
\beta_{+}^{\prime}\left(v^{2}\right)(h)=\int_{\Omega} \frac{-\Delta_{p} v-\Delta v}{v} h d z \quad \text { for all } h \in W_{0}^{1, p}(\Omega) .
$$

Since $\beta_{+}$is convex, its Gâteaux derivative is monotone, and so, from (43) and (44), we have

$$
\begin{aligned}
0 & \leq\left\langle\beta_{+}^{\prime}\left(u^{2}\right)-\beta_{+}^{\prime}\left(v^{2}\right), u^{2}-v^{2}\right\rangle_{L^{1}} \\
& =\int_{\Omega}\left(\frac{-\Delta_{p} u-\Delta u}{u}-\frac{-\Delta_{p} v-\Delta v}{v}\right)\left(u^{2}-v^{2}\right) d z \\
& =\int_{\Omega}\left(\frac{c_{5} u-c_{6} u^{p-1}}{u}-\frac{c_{5} v-c_{6} v^{p-1}}{v}\right)\left(u^{2}-v^{2}\right) d z \\
& =c_{6} \int_{\Omega}\left(v^{p-1}-u^{p-1}\right)\left(u^{2}-v^{2}\right) d z \leq 0 .
\end{aligned}
$$


Therefore $u=v$, showing that $u_{*} \in \operatorname{int} C_{+}$is the unique nontrivial positive solution of (37).

Since (37) is odd, we conclude that $v_{*}=-u_{*} \in-\operatorname{int} C_{+}$is the unique nontrivial negative solution of (37).

Having this proposition, we can now establish the existence of extremal nontrivial constant sign solutions for problem (1).

Proposition 8. If hypotheses $\mathrm{H}$ hold, then problem (1) has a smallest nontrivial positive solution $u_{+} \in \operatorname{int} C_{+}$and a biggest nontrivial negative solution $v_{-} \in-\operatorname{int} C_{+}$.

Proof. Recall that the set of nontrivial positive solutions of (1) is downward directed (i.e., if $u_{1}, u_{2}$ are nontrivial positive solutions of (1), then there exists a nontrivial positive solution $u$ of (1) such that $u \leq u_{1}$ and $u \leq u_{2}$; see [Filippakis et al. 2009, Lemma 4.2 and Proposition 4.4]). So, in order to produce the smallest nontrivial positive solution of (1), it suffices to consider the set

$$
S_{+}=\left\{u \in W_{0}^{1, p}(\Omega): u \text { is a nontrivial solution of }(1), u \in\left[0, w_{+}\right]\right\} .
$$

From Proposition 5, we know that $S_{+}$is nonempty and $S_{+} \subseteq \operatorname{int} C_{+}$.

Let $\bar{u} \in S_{+}$and consider the Carathéodory function

$$
e_{+}(z, x)=\left\{\begin{array}{cl}
0 & \text { if } x<0, \\
c_{5} x-c_{6} x^{p-1} & \text { if } 0 \leq x \leq \bar{u}(z), \\
c_{5} \bar{u}(z)-c_{6} \bar{u}(z)^{p-1} & \text { if } \bar{u}(z)<x .
\end{array}\right.
$$

We set $E_{+}(z, x)=\int_{0}^{x} e_{+}(z, s) d s$ and consider the $C^{1}$-functional $\sigma_{+}: W_{0}^{1, p}(\Omega) \rightarrow \mathbb{R}$ defined by

$$
\sigma_{+}(u)=\frac{1}{p}\|D u\|_{p}^{p}+\frac{1}{2}\|D u\|_{2}^{2}-\int_{\Omega} E_{+}(z, u(z)) d z \quad \text { for all } u \in W_{0}^{1, p}(\Omega) .
$$

It is clear from (45) that $\sigma_{+}(\cdot)$ is coercive. Also, it is sequentially weakly lower semicontinuous. So, by the Weierstrass theorem, we can find $\tilde{u} \in W_{0}^{1, p}(\Omega)$ such that

$$
\sigma_{+}(\tilde{u})=\inf \left[\sigma_{+}(u): u \in W_{0}^{1, p}(\Omega)\right] .
$$

As before (see the proof of Proposition 7), since $c_{5}>\hat{\lambda}_{1}(2)$ and $p>2$, for $t \in(0,1)$ small we have $\sigma_{+}\left(t \tilde{u}_{1,2}\right)<0$, and therefore $\sigma_{+}(\tilde{u})<0=\sigma_{+}(0)$; hence $\tilde{u} \neq 0$. From (46) this implies $\sigma_{+}^{\prime}(\tilde{u})=0$; therefore

$$
A_{p}(\tilde{u})+A(\tilde{u})=N_{e_{+}}(\tilde{u}) .
$$

On (47) we act with $-\tilde{u}^{-} \in W_{0}^{1, p}(\Omega)$ and obtain $\tilde{u} \geq 0, \tilde{u} \neq 0$ (see (45)). Also 
on (47) we act with $(\tilde{u}-\bar{u})^{+} \in W_{0}^{1, p}(\Omega)$. We obtain

$$
\begin{aligned}
& \left\langle A_{p}(\tilde{u}),(\tilde{u}-\bar{u})^{+}\right\rangle+\left\langle A(\tilde{u}),(\tilde{u}-\bar{u})^{+}\right\rangle \\
& =\int_{\Omega} e_{+}(z, \tilde{u})(\tilde{u}-\bar{u})^{+} d z=\int_{\Omega}\left(c_{5} \bar{u}-c_{6} \bar{u}^{p-1}\right)(\tilde{u}-\bar{u})^{+} d z \quad(\text { see }(45)) \\
& \leq \int_{\Omega} f(z, \bar{u})(\tilde{u}-\bar{u})^{+} d z=\left\langle A_{p}(\bar{u}),(\tilde{u}-\bar{u})^{+}\right\rangle+\left\langle A(\bar{u}),(\tilde{u}-\bar{u})^{+}\right\rangle ;
\end{aligned}
$$

this implies

$$
\int_{\{\tilde{u}>\bar{u}\}}\left(\|D \tilde{u}\|^{p-2} D \tilde{u}-\|D \bar{u}\|^{p-2} D \bar{u}, D \tilde{u}-D \bar{u}\right)_{\mathbb{R}^{N}} d z+\left\|D(\tilde{u}-\bar{u})^{+}\right\|_{2}^{2} \leq 0,
$$

and so $\tilde{u} \leq \bar{u}$.

So, we have proved that

$$
\tilde{u} \in[0, \bar{u}]=\left\{u \in W_{0}^{1, p}(\Omega): 0 \leq \tilde{u}(z) \leq \bar{u}(z) \text { a.e. in } \Omega\right\}, \quad \tilde{u} \neq 0 .
$$

From (45) and (47) it follows that

$-\Delta_{p} \tilde{u}(z)-\Delta \tilde{u}(z)=c_{5} \tilde{u}(z)-c_{6} \tilde{u}(z)^{p-1} \quad$ a.e. in $\Omega,\left.\quad \tilde{u}\right|_{\partial \Omega}=0, \quad \tilde{u} \geq 0, \quad \tilde{u} \neq 0$, whence $\tilde{u}=u_{*}$ by Proposition 7, and therefore $\tilde{u} \leq \bar{u}$.

Since $\bar{u} \in S_{+}$is arbitrary, we conclude that

$$
u_{*} \leq u \quad \text { for all } u \in S_{+} .
$$

Now let $C \subseteq S_{+}$be a chain (i.e., a totally ordered subset of $S_{+}$). Then we can find $\left\{u_{n}\right\}_{n \geq 1} \subseteq C$ such that $\inf C=\inf _{n \geq 1} u_{n}$; (see [Dunford and Schwartz 1958, p. 336]).

We have

$$
A_{p}\left(u_{n}\right)+A\left(u_{n}\right)=N_{f}\left(u_{n}\right), \quad u_{n} \in\left[u_{*}, w_{+}\right] \text {for all } n \geq 1
$$

by (48), so $\left\{u_{n}\right\}_{n \geq 1} \subseteq W_{0}^{1, p}(\Omega)$ is bounded.

So, we may assume that

$$
u_{n} \stackrel{w}{\longrightarrow} u \text { in } W_{0}^{1, p}(\Omega) \text { and } u_{n} \rightarrow u \text { in } L^{p}(\Omega) .
$$

On (49) we act with $u_{n}-u \in W_{0}^{1, p}(\Omega)$, pass to the limit as $n \rightarrow \infty$ and use (50). Then

$$
\lim _{n \rightarrow \infty}\left(\left\langle A_{p}\left(u_{n}\right), u_{n}-u\right\rangle+\left\langle A\left(u_{n}\right), u_{n}-u\right\rangle\right)=0,
$$

and so (reasoning as in Claim 1 in the proof of Proposition 6)

$$
u_{n} \rightarrow u \quad \text { in } W_{0}^{1, p}(\Omega) .
$$


So, if in (49) we pass to the limit as $n \rightarrow \infty$ and use (51), then (48) yields

$$
A_{p}(u)+A(u)=N_{f}(u), \quad u_{*} \leq u,
$$

which leads to $u \in S_{+}, u=\inf C$.

Because $C$ is an arbitrary chain, the Kuratowski-Zorn lemma gives the existence of a minimal element $u_{+} \in S_{+}$of $S_{+}$. But recall that $S_{+}$is downward directed. So, if $u \in S_{+}$, we can find $y \in S_{+}$such that $y \leq u, y \leq u_{+}$. The minimality of $u_{+}$ implies that $u_{+}=y$ and so $u_{+} \leq u$. Since $u \in S_{+}$is arbitrary, we conclude that $u_{+}$ is the smallest nontrivial positive solution of (1).

Similarly, let $S_{-}$be the set of nontrivial negative solutions of (1) in $\left[w_{-}, 0\right]$. Then $S_{-}$is upward directed (i.e., if $v_{1}, v_{2} \in S_{-}$, then we can find $v \in S_{-}$such that $v_{1} \leq v, v_{2} \leq v$; see [Filippakis et al. 2009, Lemma 4.3]). Let $\bar{v} \in S_{-}$and consider the Carathéodory function

$$
e_{-}(z, x)=\left\{\begin{array}{cl}
c_{5} \bar{v}(z)-c_{6}|\bar{v}(z)|^{p-2} \bar{v}(z) & \text { if } x<\bar{v}(z), \\
c_{5} x-c_{6}|x|^{p-2} x & \text { if } \bar{v}(z) \leq x \leq 0, \\
0 & \text { if } 0<x
\end{array}\right.
$$

We set $E_{-}(z, x)=\int_{0}^{x} e_{-}(z, s) d s$ and consider the $C^{1}$-functional $\sigma_{-}: W_{0}^{1, p}(\Omega) \rightarrow \mathbb{R}$ defined by

$$
\sigma_{-}(u)=\frac{1}{p}\|D u\|_{p}^{p}+\frac{1}{2}\|D u\|_{2}^{2}-\int_{\Omega} E_{-}(z, u(z)) d z \quad \text { for all } u \in W_{0}^{1, p}(\Omega) .
$$

Reasoning as above, we produce $v_{-} \in-\operatorname{int} C_{+}$, the smallest nontrivial negative solution of (1).

To implement the strategy outlined in the beginning of this section and produce a nodal solution, we need to be able to identify the nonzero critical points of $\varphi$ distinct from $u_{*}$ and $v_{*}$ which are in the order interval $\left[v_{*}, u_{*}\right]$. This can be done using critical groups. For this reason, we compute the critical groups of $\varphi$ at the origin.

Proposition 9. If hypotheses $\mathrm{H}$ hold, then $C_{k}(\varphi, 0)=\delta_{k, d_{m}} \mathbb{Z}$ for all $k \geq 0$ with $d_{m}=\operatorname{dim} \bigoplus_{i=1}^{m} E\left(\hat{\lambda}_{i}(2)\right)$.

Proof. Let $\mu \in\left(\hat{\lambda}_{m}(2), \hat{\lambda}_{m+1}(2)\right)$ and consider the $C^{2}$-functional $\varphi_{0}: W_{0}^{1, p}(\Omega) \rightarrow \mathbb{R}$ defined by

$$
\varphi_{0}(u)=\frac{1}{p}\|D u\|_{p}^{p}+\frac{1}{2}\|D u\|_{2}^{2}-\frac{\mu}{2}\|u\|_{2}^{2} \quad \text { for all } u \in W_{0}^{1, p}(\Omega) .
$$

We consider the homotopy $h_{0}:[0,1] \times W_{0}^{1, p}(\Omega) \rightarrow \mathbb{R}$ defined by

$$
h_{0}(t, u)=t \varphi(u)+(1-t) \varphi_{0}(u) \quad \text { for all }(t, u) \in[0,1] \times W_{0}^{1, p}(\Omega) .
$$


Clearly $h_{0}(0, \cdot)=\varphi_{0}(\cdot)$ and $h_{1}(0, \cdot)=\varphi(\cdot)$.

It is easy to see that, since $p>2, \varphi_{0}$ satisfies the $\mathrm{C}$-condition. Also, reasoning as in Claim 1 in the proof of Proposition 6, via hypothesis H(iv), we show that $\varphi$ satisfies the C-condition.

Suppose we can find $\left\{t_{n}\right\}_{n \geq 1} \subseteq[0,1]$ and $\left\{u_{n}\right\}_{n \geq 1} \subseteq W_{0}^{1, p}(\Omega)$ such that

$$
t_{n} \rightarrow t, u_{n} \rightarrow 0 \text { in } W_{0}^{1, p}(\Omega) \text { and } \quad\left(h_{0}\right)_{u}^{\prime}\left(t_{n}, u_{n}\right)=0 \text { for all } n \geq 1 .
$$

From the equality in (52), we have

$$
A_{p}\left(u_{n}\right)+A\left(u_{n}\right)=t_{n} N_{f}\left(u_{n}\right)+\left(1-t_{n}\right) \mu u_{n},
$$

and therefore

(53) $-\Delta_{p} u_{n}(z)-\Delta u_{n}(z)=t_{n} f\left(z, u_{n}(z)\right)+\left(1-t_{n}\right) \mu u_{n}(z)$ a.e. in $\Omega,\left.\quad u_{n}\right|_{\partial \Omega}=0$.

Since $\mu \in\left(\hat{\lambda}_{m}(2), \hat{\lambda}_{m+1}(2)\right)$, we have

$$
t f(z, x) x+(1-t) \mu x^{2} \geq c_{5} x^{2}-c_{6}|x|^{p} \quad \text { for a.a. } z \in \Omega \text {, all } x \in \mathbb{R}, t \in[0,1],
$$

where $c_{5}>\hat{\lambda}_{1}(2)$ and $c_{6}>0$ are as before (see (37)). Then from (53) and the proof of Proposition 8, we have $u_{*} \leq u_{n}$ for all $n \geq 1$, which contradicts (52). Therefore (52) cannot happen and so the homotopy invariance of critical groups (see, for example, [Chang 2005]) implies that $C_{k}\left(h_{0}(0, \cdot), 0\right)=C_{k}\left(h_{0}(1, \cdot), 0\right)$ for all $k \geq 0$, whence

$$
C_{k}\left(\varphi_{0}, 0\right)=C_{k}(\varphi, 0) \quad \text { for all } k \geq 0 .
$$

Note that $\varphi_{0}^{\prime \prime}(0)=A-\mu I$ (see [Cingolani and Vannella 2003, p. 277]) and recall that $\mu \in\left(\hat{\lambda}_{m}(2), \hat{\lambda}_{m+1}(2)\right)$. Invoking Theorem 1.1 of [Cingolani and Vannella 2003], we have $C_{k}\left(\varphi_{0}, 0\right)=\delta_{k, d_{m}} \mathbb{Z}$ for all $k \geq 0$, with $d_{m}=\operatorname{dim} \bigoplus_{i=1}^{m} E\left(\hat{\lambda}_{i}(2)\right)$. Using (54) concludes the proof.

Now we have all the necessary tools to complete our strategy and produce a nodal solution.

Proposition 10. If hypotheses $\mathrm{H}$ hold, problem (1) has a nodal solution $y_{0} \in C_{0}^{1}(\bar{\Omega})$ such that

$$
u_{+}-y_{0} \in \operatorname{int} C_{+} \quad \text { and } \quad y_{0}-v_{-} \in \operatorname{int} C_{+} .
$$

Proof. Let $u_{+} \in \operatorname{int} C_{+}$and $v_{-} \in-\operatorname{int} C_{+}$be the two extremal nontrivial constant sign solutions of (1) produced in Proposition 8. Using these two solutions, we introduce the following truncation of the reaction $f(z, \cdot)$ :

$$
g(z, x)=\left\{\begin{array}{cl}
f\left(z, v_{-}(z)\right) & \text { if } x<v_{-}(z) \\
f(z, x) & \text { if } v_{-}(z) \leq x \leq u_{+}(z), \\
f\left(z, u_{+}(z)\right) & \text { if } u_{+}(z)<x
\end{array}\right.
$$


This is a Carathéodory function. We set $G(z, x)=\int_{0}^{x} g(z, s) d s$. Also, let $g_{ \pm}(z, x)=g\left(z, \pm x^{ \pm}\right)$and $G_{ \pm}(z, x)=\int_{0}^{x} g_{ \pm}(z, s) d s$. Then we introduce the $C^{1}$-functionals $\xi^{*}, \xi_{ \pm}^{*}: W_{0}^{1, p}(\Omega) \rightarrow \mathbb{R}$ defined by

$$
\begin{aligned}
& \xi^{*}(u)=\frac{1}{p}\|D u\|_{p}^{p}+\frac{1}{2}\|D u\|_{2}^{2}-\int_{\Omega} G(z, u(z)) d z, \\
& \xi_{ \pm}^{*}(u)=\frac{1}{p}\|D u\|_{p}^{p}+\frac{1}{2}\|D u\|_{2}^{2}-\int_{\Omega} G_{ \pm}(z, u(z)) d z \quad \text { for all } u \in W_{0}^{1, p}(\Omega) .
\end{aligned}
$$

Claim 1. $K_{\xi^{*}} \subseteq\left[v_{-}, u_{+}\right], K_{\xi_{+}^{*}}=\left\{0, u_{+}\right\}, K_{\xi_{-}^{*}}=\left\{0, v_{-}\right\}$.

Proof. Let $u \in K_{\xi^{*}}$. Then we have

$$
A_{p}(u)+A(u)=N_{g}(u) .
$$

On (56) we act with $\left(u-u_{+}\right)^{+} \in W_{0}^{1, p}(\Omega)$ and obtain

$$
\begin{aligned}
& \left\langle A_{p}(u),\left(u-u_{+}\right)^{+}\right\rangle+\left\langle A(u),\left(u-u_{+}\right)^{+}\right\rangle \\
& =\int_{\Omega} g(z, u)\left(u-u_{+}\right)^{+} d z=\int_{\Omega} f\left(z, u_{+}\right)\left(u-u_{+}\right)^{+} d z \quad \text { (see (55)) } \\
& =\left\langle A_{p}\left(u_{+}\right),\left(u-u_{+}\right)^{+}\right\rangle+\left\langle A\left(u_{+}\right),\left(u-u_{+}\right)^{+}\right\rangle,
\end{aligned}
$$

so that

$\int_{\left\{u>u_{+}\right\}}\left(\|D u\|^{p-2} D u-\left\|D u_{+}\right\|^{p-2} D u_{+}, D u-D u_{+}\right)_{\mathbb{R}^{N}} d z+\left\|D\left(u-u_{+}\right)^{+}\right\|_{2}^{2}=0$ and therefore $u \leq u_{+}$.

Similarly, acting on (56) with $\left(v_{-}-u\right)^{+} \in W_{0}^{1, p}(\Omega)$, we show that $v_{-} \leq u$. Therefore $K_{\xi^{*}} \subseteq\left[v_{-}, u_{+}\right]$.

In a similar fashion, we show that $K_{\xi_{+}^{*}} \subseteq\left[0, u_{+}\right]$. Clearly $\left\{0, u_{+}\right\} \subseteq K_{\xi_{+}^{*}}$. The extremality of $u_{+}$implies that $K_{\xi_{+}^{*}}=\left\{0, u_{+}\right\}$. Similarly, $K_{\xi_{-}^{*}}=\left\{v_{-}, 0\right\}$. This proves Claim 1.

Claim 2. The functions $u_{+} \in \operatorname{int} C_{+}$and $v_{-} \in-\operatorname{int} C_{+}$are both local minimizers of the functional $\xi^{*}$.

Proof. It is clear from (55) that $\xi_{+}^{*}$ is coercive. Also, it is sequentially weakly lower semicontinuous. So, we can find $\tilde{u} \in W_{0}^{1, p}(\Omega)$ such that

$$
\xi_{+}^{*}(\tilde{u})=\inf \left[\xi_{+}^{*}(u): u \in W_{0}^{1, p}(\Omega)\right] .
$$

As in the proof of Proposition 5, using hypothesis H(vii) and the fact that $2<p$, we have $\xi_{+}^{*}\left(t \hat{u}_{1,2}\right)<0$ for $t \in(0,1)$ small, which give $\xi_{+}^{*}(\tilde{u})<0=\xi_{+}^{*}(0)$; hence $\tilde{u} \neq 0$, showing that $\tilde{u}=u_{+}$by Claim 1 . 
But $u_{+} \in \operatorname{int} C_{+}$and $\left.\xi^{*}\right|_{C_{+}}=\left.\xi_{+}^{*}\right|_{C_{+}}$(see (55)). Therefore $u_{+}$is a local $C_{0}^{1}(\bar{\Omega})$ minimizer of $\xi^{*}$; hence it is a local $W_{0}^{1, p}(\Omega)$-minimizer of $\xi^{*}$ (see Theorem 2). Similarly for $v_{-} \in-$ int $C_{+}$, using this time the functional $\xi_{-}^{*}$.

We assume that $K_{\xi *}$ is finite (otherwise, we already have an infinity of distinct nodal solutions). Also, without any loss of generality, we assume that $\xi^{*}\left(v_{-}\right) \leq \xi_{+}^{*}\left(u_{+}\right)$(the analysis is similar if the opposite inequality holds). By virtue of Claim 2, as in [Aizicovici et al. 2008, proof of Proposition 29] we can find $\rho \in(0,1)$ small such that

$$
\xi^{*}\left(v_{-}\right) \leq \xi^{*}\left(u_{+}\right)<\inf \left[\xi^{*}(u):\left\|u-u_{+}\right\|=\rho\right]=\eta_{\rho}^{*} \quad \text { and } \quad\left\|v_{-}-u_{+}\right\|>\rho .
$$

Note that $\xi^{*}$ is coercive (see (55)); hence it satisfies the C-condition. This fact and (57) permit the use of the mountain pass theorem. So, we can find $y_{0} \in W_{0}^{1, p}(\Omega)$ such that

$$
y_{0} \in K_{\xi^{*}} \quad \text { and } \quad \eta_{\rho}^{*} \leq \xi^{*}\left(y_{0}\right) .
$$

From (57), (58) and Claim 1, we have

$$
y_{0} \in\left[v_{-}, u_{+}\right], \quad y_{0} \notin\left\{v_{-}, u_{+}\right\} .
$$

Since $y_{0}$ is a critical point of $\xi^{*}$ of mountain pass type, we have

$$
C_{1}\left(\xi^{*}, y_{0}\right) \neq 0 \text {. }
$$

Using the homotopy invariance of critical groups, we have $C_{k}\left(\xi^{*}, 0\right)=C_{k}(\varphi, 0)$ for all $k \geq 0$, which gives (see Proposition 9)

$$
C_{k}\left(\xi^{*}, 0\right)=\delta_{k, d_{m}} \mathbb{Z} \quad \text { for all } k \geq 0 .
$$

From (60), (61) and since $d_{m} \geq 2$, we infer that $y_{0} \neq 0$. Then the extremality of $u_{+}$and $v_{-}$and the fact that $y_{0} \in\left[v_{-}, u_{+}\right]$imply that $y_{0} \in C_{0}^{1}(\bar{\Omega})$ (see [Lieberman 1991]) is a nodal solution of (1).

Using the tangency principle of [Pucci and Serrin 2007, p. 35], we have

$$
v_{-}(z)<y_{0}(z)<u_{+}(z) \text { for all } z \in \Omega \text {. }
$$

Let $\rho=\max \left\{\left\|u_{+}\right\|,\left\|v_{-}\right\|\right\}$and let $\xi_{\rho}>0$ as postulated by hypothesis $\mathrm{H}(\mathrm{vi})$. Then, for $\tilde{\xi}>\xi_{\rho}$, we have

$$
\begin{aligned}
-\Delta_{p} y_{0}(z)-\Delta y_{0}(z) & +\tilde{\xi}\left|y_{0}(z)\right|^{p-2} y_{0}(z) \\
= & f\left(z, y_{0}(z)\right)+\tilde{\xi}\left|y_{0}(z)\right|^{p-2} y_{0}(z) \leq f\left(z, u_{+}(z)\right)+\tilde{\xi} u_{+}(z)^{p-1} \\
= & -\Delta_{p} u_{+}(z)-\Delta u_{+}(z)+\tilde{\xi} u_{+}(z)^{p-1} \quad \text { a.e. in } \Omega .
\end{aligned}
$$


Setting $h(z)=f\left(z, y_{0}(z)\right)+\tilde{\xi}\left|y_{0}(z)\right|^{p-2} y_{0}(z)$ and $\hat{h}=f\left(z, u_{+}(z)\right)+\tilde{\xi} u_{+}(z)^{p-1}$, we see that $h, \hat{h} \in L^{\infty}(\Omega)$ and $h \prec \hat{h}$. Since $u_{+} \in \operatorname{int} C_{+}$, we can apply Proposition 3 and infer that $u_{+}-y_{0} \in \operatorname{int} C_{+}$. Similarly we show that $y_{0}-v_{-} \in \operatorname{int} C_{+}$.

So, we can state the following multiplicity theorem concerning problem (1). We stress that the result is proved without assuming any differentiability on the function $x \rightarrow f(z, x)$ (see hypotheses $\mathrm{H}$ ). In addition our multiplicity theorem provides precise sign information for all the solutions produced.

Theorem 11. If hypotheses $\mathrm{H}$ hold, the problem (1) has at least five nontrivial smooth solutions:

$$
\begin{aligned}
& u_{0}, \hat{u} \in \operatorname{int} C_{+} \quad \text { with } u_{0}-\hat{u} \in \operatorname{int} C_{+}, \\
& v_{0}, \hat{v} \in-\operatorname{int} C_{+} \quad \text { with } v_{0}-\hat{v} \in \operatorname{int} C_{+},
\end{aligned}
$$

and

$$
y_{0} \in C_{0}^{1}(\bar{\Omega}) \quad \text { nodal s.t. } \quad y_{0}-v_{0} \in \operatorname{int} C_{+}, \quad u_{0}-y_{0} \in \operatorname{int} C_{+} .
$$

Next, by strengthening the regularity condition on $f(z, \cdot)$, we will be able to produce a second nodal solution.

The new hypotheses on the reaction $f(z, x)$ are the following:

Hypotheses $\widehat{\mathbf{H}}$. (i) $f: \Omega \times \mathbb{R} \rightarrow \mathbb{R}$ is a measurable function.

(ii) For a.a. $z \in \Omega$, we have $f(z, 0)=0$ and $f(z, \cdot) \in C^{1}(\mathbb{R})$.

(iii) $\left|f_{x}^{\prime}(z, x)\right| \leq \alpha(z)+c|x|^{r-2}$ for a.a. $z \in \Omega$, all $x \in \mathbb{R}$, with $\alpha \in L^{\infty}(\Omega)_{+}, c>0$ and $p \leq r<p^{*}$.

(iv) If $F(z, x)=\int_{0}^{x} f(z, s) d s$, then

$$
\lim _{x \rightarrow \pm \infty} \frac{p F(z, x)}{|x|^{p}}=\hat{\lambda}_{1}(p) \quad \text { uniformly for a.a. } z \in \Omega
$$

and, for some $\tau>2$,

$$
\lim _{x \rightarrow \pm \infty} \frac{f(z, x) x-p F(z, x)}{|x|^{\tau}} \leq \hat{\beta}<0 \quad \text { uniformly for a.a. } z \in \Omega .
$$

(v) There exist functions $w_{ \pm} \in W^{1, p}(\Omega) \cap C(\bar{\Omega})$ such that

$$
\begin{aligned}
& w_{-}(z) \leq c_{-}<0<c_{+} \leq w_{+}(z) \quad \text { for all } z \in \bar{\Omega}, \\
& \underset{\Omega}{\operatorname{ess} \sup } f\left(\cdot, w_{+}(\cdot)\right) \leq 0 \leq \underset{\Omega}{\operatorname{essinf}} f\left(\cdot, w_{-}(\cdot)\right)
\end{aligned}
$$

and

$$
\begin{aligned}
& A_{p}\left(w_{-}\right)+A\left(w_{-}\right) \leq 0 \leq A_{p}\left(w_{+}\right)+A\left(w_{+}\right) \text {in } W^{-1, p^{\prime}}(\Omega)=W_{0}^{1, p}(\Omega)^{*} \\
& \text { (where } \left.1 / p+1 / p^{\prime}=1\right) .
\end{aligned}
$$


(vi) For every $\rho>0$, there exists $\xi_{\rho}>0$ such that, for a.a. $z \in \Omega$, the function $x \rightarrow f(z, x)+\xi_{\rho}|x|^{p-2} x$ is nondecreasing on $[-\rho, \rho]$.

(vii) There exists integer $m \geq 2$ such that

$$
\begin{gathered}
\hat{\lambda}_{m}(2) \leq f_{x}^{\prime}(z, 0) \leq \hat{\lambda}_{m+1}(2) \quad \text { a.e. in } \Omega, \quad \hat{\lambda}_{m}(2) \neq f_{x}^{\prime}(z, \cdot), \quad \hat{\lambda}_{m+1}(2) \neq f_{x}^{\prime}(z, \cdot), \\
f_{x}^{\prime}(z, 0)=\lim _{x \rightarrow 0} \frac{f(z, x)}{x} \quad \text { uniformly for a.a. } z \in \Omega .
\end{gathered}
$$

In what follows, we use the notation and the functionals introduced in the proof of Proposition 10.

Proposition 12. If hypotheses $\widehat{\mathrm{H}}$ hold, then problem (1) has a second nodal solution $\hat{y} \in C_{0}^{1}(\bar{\Omega})$ such that

$$
u_{+}-\hat{y} \in \operatorname{int} C_{+} \quad \text { and } \quad \hat{y}-v_{-} \in \operatorname{int} C_{+} .
$$

Proof. We assume that $K_{\xi_{+}^{*}}$ is finite (otherwise we already have an infinity of nodal solutions). From the proof of Proposition 10, we have

$$
\left\{0, u_{+}, v_{-}, y_{0}\right\} \subseteq K_{\xi_{+}^{*}} \subseteq\left[v_{-}, u_{+}\right] .
$$

We know that $u_{+} \in \operatorname{int} C_{+}$and $v_{-} \in-\operatorname{int} C_{+}$are local minimizers of the functional $\xi_{*}$ (see Claim 2 in the proof of Proposition 10). So, we have

$$
C_{k}\left(\xi^{*}, u_{+}\right)=C_{k}\left(\xi^{*}, v_{-}\right)=\delta_{k, 0} \mathbb{Z} \quad \text { for all } k \geq 0 .
$$

Also, from (61) we have

$$
C_{k}\left(\xi^{*}, 0\right)=\delta_{k, d_{m}} \mathbb{Z} \quad \text { for all } k \geq 0 .
$$

Moreover, since $\xi^{*}$ is coercive (see (55)), we have

$$
C_{k}\left(\xi^{*}, \infty\right)=\delta_{k, 0} \mathbb{Z} \quad \text { for all } k \geq 0 .
$$

Claim 1. $C_{k}\left(\varphi, y_{0}\right)=C_{k}\left(\xi^{*}, y_{0}\right)$ for all $k \geq 0$.

Proof. We consider the homotopy $\tilde{h}:[0,1] \times W_{0}^{1, p}(\Omega) \rightarrow \mathbb{R}$ defined by

$$
\tilde{h}(t, u)=(1-t) \xi^{*}(u)+t \varphi(u) \quad \text { for all }(t, u) \in[0,1] \times W_{0}^{1, p}(\Omega) .
$$

We have $\tilde{h}(0, \cdot)=\xi^{*}(\cdot)$ and $\tilde{h}(1, \cdot)=\varphi(\cdot)$ and both functionals satisfy the C-condition. Let $\left\{t_{n}\right\}_{n \geq 1} \subseteq[0,1]$ and $\left\{u_{n}\right\}_{n \geq 1} \subseteq W_{0}^{1, p}(\Omega)$ such that

$$
t_{n} \rightarrow t, u_{n} \rightarrow y_{0} \text { in } W_{0}^{1, p}(\Omega) \quad \text { and } \quad(\tilde{h})_{u}^{\prime}\left(t_{n}, u_{n}\right)=0 \quad \text { for all } n \geq 1 .
$$

From the equation in (66), we have

$$
A_{p}\left(u_{n}\right)+A\left(u_{n}\right)=\left(1-t_{n}\right) N_{g}\left(u_{n}\right)+t_{n} N_{f}\left(u_{n}\right) \text { for all } n \geq 1 \text {, }
$$


by (55). Hence

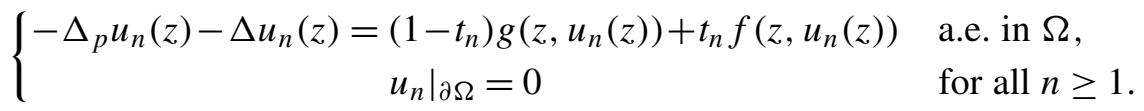

From (67) and [Lieberman 1991], we know that we can find $\gamma \in(0,1)$ and $M>0$ such that

$$
u_{n} \in C_{0}^{1, \gamma}(\bar{\Omega}) \quad \text { and } \quad\left\|u_{n}\right\|_{C_{0}^{1, \gamma}(\bar{\Omega})} \leq M \quad \text { for all } n \geq 1 .
$$

From (68) and the compact embedding of $C_{0}^{1, \gamma}(\bar{\Omega})$ into $C_{0}^{1}(\bar{\Omega})$, by passing to a suitable subsequence if necessary, we may assume by (66) that

$$
u_{n} \rightarrow y_{0} \quad \text { in } C^{1}(\bar{\Omega}) \text {. }
$$

Since $y_{0} \in \operatorname{int}_{C_{0}^{1, \gamma}(\bar{\Omega})}\left[v_{-}, u_{+}\right]$(see Theorem 11), from (69) it follows that

$$
u_{n} \in\left[v_{-}, u_{+}\right], \quad u_{n} \neq v_{-}, \quad u_{n} \neq u_{+} \quad \text { for all } n \geq n_{0} ;
$$

this, by (55), gives $\left\{u_{n}\right\}_{n \geq n_{0}} \subseteq K_{\xi^{*}}$, which contradicts our hypothesis that $K_{\xi^{*}}$ is finite. So, (66) cannot happen, and, from the homotopy invariance of critical groups, we have

$$
C_{k}\left(\tilde{h}(0, \cdot), y_{0}\right)=C_{k}\left(\tilde{h}(1, \cdot), y_{0}\right) \quad \text { for all } k \geq 0,
$$

which yields the claim.

From (60) and Claim 1, we have

$$
C_{1}\left(\varphi, y_{0}\right) \neq 0 .
$$

Claim 2. $C_{k}\left(\varphi, y_{0}\right)=\delta_{k, 1} \mathbb{Z}$ for all $k \geq 0$.

Proof. From [Cingolani and Vannella 2007, Lemma 2.2], we know that we can find $\rho>0$ and a $C^{2}$-function $\vartheta: V \cap \bar{B}_{\rho} \rightarrow \mathbb{R}$ (recall $V=H^{-} \oplus H^{0}$ (see Section 2), while $\left.\bar{B}_{\rho}=\left\{u \in W_{0}^{1, p}(\Omega):\|u\| \leq \rho\right\}\right)$ such that

$$
\left\langle\vartheta^{\prime \prime}(0) v, u\right\rangle=\left\langle\varphi^{\prime \prime}\left(y_{0}\right) v, u\right\rangle \quad \text { for all } u, v \in W_{0}^{1, p}(\Omega) .
$$

In addition $\vartheta^{\prime \prime}(0)$ is a Fredholm operator and $\operatorname{ker} \vartheta^{\prime \prime}(0)=H^{0}$. From [Cingolani and Vannella 2003, p. 286], we have

$$
C_{k}\left(\varphi, y_{0}\right)=C_{k}(\vartheta, 0) \quad \text { for all } k \geq 0 .
$$

Then (70), (71) imply that

$$
C_{1}(\vartheta, 0) \neq 0
$$

and so

$$
d_{-}=\operatorname{dim} H^{-} \leq 1
$$


Let $d_{0}=\operatorname{dim} H^{0}$. We consider two cases:

Case I: $d_{0}=0$. In this case, $u=0$ is a nondegenerate critical point of $\vartheta$ with Morse index $d_{-}$. Hence $C_{k}(\vartheta, 0)=\delta_{k, d_{-}} \mathbb{Z}$ for all $k \geq 0$ (see Section 2). In view of (72) we then have $d_{-}=1$, so $C_{k}\left(\varphi, y_{0}\right)=\delta_{k, 1} \mathbb{Z}$ for all $k \geq 0$ (see (71)).

Case II: $d_{0}>0$. In this case, $u=0$ is a degenerate critical point of $\vartheta$. From (73) we see that $d_{-}=1$ or $d_{-}=0$.

If $d_{-}=1$, then, from [Cingolani and Vannella 2003, p. 286], we have $C_{k}(\vartheta, 0)=$ $\delta_{k, 1} \mathbb{Z}$ for all $k \geq 0$, so $C_{k}\left(\varphi, y_{0}\right)=\delta_{k, 1} \mathbb{Z}$ for all $k \geq 0$ (see (71)).

If $d_{-}=0$, then, from (72) and [Cingolani and Degiovanni 2009], we have $C_{k}(\vartheta, 0)=\delta_{k, 1} \mathbb{Z}$ for all $k \geq 0$, so $C_{k}\left(\varphi, y_{0}\right)=\delta_{k, 1} \mathbb{Z}$ for all $k \geq 0($ see $(71))$.

This proves Claim 2.

Claims 1 and 2 imply that

$$
C_{k}\left(\xi^{*}, y_{0}\right)=\delta_{k, 1} \mathbb{Z} \quad \text { for all } k \geq 0 .
$$

Suppose that $K_{\xi_{+}^{*}}=\left\{0, u_{+}, v_{-}, y_{0}\right\}$. Then, from (63), (64), (65), (74) and the Morse relation (see (4)) with $t=-1$, we have $(-1)^{d_{m}}=0$, a contradiction. Therefore, we can find $\hat{y} \in K_{\xi_{+}^{*}}, \hat{y} \notin\left\{0, u_{+}, v_{-}, y_{0}\right\}$. We have $\hat{y} \in\left[v_{-}, u_{+}\right]$(see Claim 1 in the proof of Proposition 10) and so $\hat{y}$ is nodal. Moreover, $\hat{y} \in C_{0}^{1}(\bar{\Omega})$ (nonlinear regularity) and, as we did for $y_{0}$ (see the proof of Proposition 10), we show that $\hat{y} \in \operatorname{int}_{C_{0}^{1}(\bar{\Omega})}\left[v_{-}, u_{+}\right]$.

Now we can state the second multiplicity theorem for problem (1).

Theorem 13. If hypotheses $\widehat{\mathrm{H}}$ hold, then problem (1) has at least six nontrivial smooth solutions

$$
\begin{aligned}
& u_{0}, \hat{u} \in \operatorname{int} C_{+} \quad \text { with } \hat{u}-u_{0} \in \operatorname{int} C_{+}, \\
& v_{0}, \hat{v} \in-\operatorname{int} C_{+} \quad \text { with } v_{0}-\hat{v} \in \operatorname{int} C_{+}
\end{aligned}
$$

and $y_{0}, \hat{y} \in C_{0}^{1}(\bar{\Omega})$ nodal with $u_{0}-y_{0}, u_{0}-\hat{y} \in \operatorname{int} C_{+}$and $y_{0}-v_{0}, \hat{y}-v_{0} \in \operatorname{int} C_{+}$.

\section{Acknowledgement}

The authors wish to thank a knowledgeable referee for helpful remarks and for providing additional references.

\section{References}

[Aizicovici et al. 2008] S. Aizicovici, N. S. Papageorgiou, and V. Staicu, "Degree theory for operators of monotone type and nonlinear elliptic equations with inequality constraints", Mem. Amer. Math. Soc. 196:915 (2008). MR 2010c:47211 Zbl 1165.47041

[Anane 1987] A. Anane, "Simplicité et isolation de la première valeur propre du $p$-Laplacien avec poids”, C. R. Acad. Sci. Paris Sér. I Math. 305:16 (1987), 725-728. MR 89e:35124 Zbl 0633.35061 
[Anane and Tsouli 1996] A. Anane and N. Tsouli, "On the second eigenvalue of the $p$-Laplacian", pp. 1-9 in Nonlinear partial differential equations (Fès, 1994), edited by A. Benkirane and J.-P. Gossez, Pitman Res. Notes Math. Ser. 343, Longman, Harlow, 1996. MR 97k:35190 Zbl 0854.35081

[Arcoya and Ruiz 2006] D. Arcoya and D. Ruiz, "The Ambrosetti-Prodi problem for the $p$-Laplace operator", Comm. Partial Differential Equations 31:4-6 (2006), 849-865. MR 2007h:35123 Zbl 1101.35033

[Bartsch et al. 2005] T. Bartsch, Z. Liu, and T. Weth, "Nodal solutions of a $p$-Laplacian equation", Proc. London Math. Soc. (3) 91:1 (2005), 129-152. MR 2006a:35072 Zbl 1162.35364

[Benci et al. 2000] V. Benci, P. D'Avenia, D. Fortunato, and L. Pisani, "Solitons in several space dimensions: Derrick's problem and infinitely many solutions", Arch. Ration. Mech. Anal. 154:4 (2000), 297-324. MR 2002c:35217 Zbl 0973.35161

[Benguria et al. 1981] R. Benguria, H. Brézis, and E. H. Lieb, "The Thomas-Fermi-von Weizsäcker theory of atoms and molecules”, Comm. Math. Phys. 79:2 (1981), 167-180. MR 83m:81114 Zbl 0478.49035

[Brézis and Nirenberg 1993] H. Brézis and L. Nirenberg, " $H^{1}$ versus $C^{1}$ local minimizers", $C . R$. Acad. Sci. Paris Sér. I Math. 317:5 (1993), 465-472. MR 94g:49044 Zbl 0803.35029

[Chang 2005] K.-C. Chang, Methods in nonlinear analysis, Springer, Berlin, 2005. MR 2007b:47169 Zbl 1081.47001

[Cingolani and Degiovanni 2005] S. Cingolani and M. Degiovanni, "Nontrivial solutions for $p$ Laplace equations with right-hand side having $p$-linear growth at infinity", Comm. Partial Differential Equations 30:7-9 (2005), 1191-1203. MR 2006k:35086 Zbl 1162.35367

[Cingolani and Degiovanni 2009] S. Cingolani and M. Degiovanni, "On the Poincaré-Hopf theorem for functionals defined on Banach spaces", Adv. Nonlinear Stud. 9:4 (2009), 679-699. MR 2010j:58027 Zbl 1187.58016

[Cingolani and Vannella 2003] S. Cingolani and G. Vannella, "Critical groups computations on a class of Sobolev Banach spaces via Morse index”, Ann. Inst. H. Poincaré Anal. Non Linéaire 20:2 (2003), 271-292. MR 2004f:35033 Zbl 1023.58004

[Cingolani and Vannella 2007] S. Cingolani and G. Vannella, "Marino-Prodi perturbation type results and Morse indices of minimax critical points for a class of functionals in Banach spaces", Ann. Mat. Pura Appl. (4) 186:1 (2007), 155-183. MR 2008b:58017 Zbl 1232.58006

[Derrick 1964] G. H. Derrick, "Comments on nonlinear wave equations as models for elementary particles”, J. Mathematical Phys. 5 (1964), 1252-1254. MR 30 \#4510

[Díaz and Saá 1987] J. I. Díaz and J. E. Saá, "Existence et unicité de solutions positives pour certaines équations elliptiques quasilinéaires”, C. R. Acad. Sci. Paris Sér. I Math. 305:12 (1987), 521-524. MR 89e:35051 Zbl 0656.35039

[Dunford and Schwartz 1958] N. Dunford and J. T. Schwartz, Linear operators, I: General theory, Pure Appl. Math. 7, Wiley Interscience, New York, 1958. MR 22 \#8302 Zbl 0084.10402

[Filippakis et al. 2009] M. Filippakis, A. Kristály, and N. S. Papageorgiou, "Existence of five nonzero solutions with exact sign for a $p$-Laplacian equation”, Discrete Contin. Dyn. Syst. 24:2 (2009), 405-440. MR 2010b:35122 Zbl 1169.35024

[Finn and Gilbarg 1957] R. Finn and D. Gilbarg, "Asymptotic behavior and uniqueness of plane subsonic flows", Comm. Pure Appl. Math. 10 (1957), 23-63. MR 19,203d Zbl 0077.18801

[García Azorero and Peral Alonso 1987] J. P. García Azorero and I. Peral Alonso, "Existence and nonuniqueness for the $p$-Laplacian: nonlinear eigenvalues", Comm. Partial Differential Equations 12:12 (1987), 1389-1430. MR 89e:35058 Zbl 0637.35069 
[García Azorero et al. 2000] J. P. García Azorero, I. Peral Alonso, and J. J. Manfredi, "Sobolev versus Hölder local minimizers and global multiplicity for some quasilinear elliptic equations", Commun. Contemp. Math. 2:3 (2000), 385-404. MR 2001k:35062 Zbl 0965.35067

[Gasiński and Papageorgiou 2006] L. Gasiński and N. S. Papageorgiou, Nonlinear analysis, Ser. Math. Anal. Appl. 9, Chapman \& Hall/CRC, Boca Raton, FL, 2006. MR 2006e:47001 Zbl 1086.47001

[Gasiński and Papageorgiou 2012] L. Gasiński and N. S. Papageorgiou, "Multiple solutions for nonlinear coercive problems with a nonhomogeneous differential operator and a nonsmooth potential", Set-Valued Var. Anal. 20:3 (2012), 417-443. MR 2949635 Zbl 06113614

[Gilbarg and Trudinger 2001] D. Gilbarg and N. S. Trudinger, Elliptic partial differential equations of second order, 2nd ed., Springer, Berlin, 2001. MR 2001k:35004 Zbl 1042.35002

[Iturriaga et al. 2010] L. Iturriaga, E. Massa, J. Sánchez, and P. Ubilla, "Positive solutions of the p-Laplacian involving a superlinear nonlinearity with zeros", J. Differential Equations 248:2 (2010), 309-327. MR 2010k:35196 Zbl 1181.35117

[Ladyzhenskaya and Ural'tseva 1968] O. A. Ladyzhenskaya and N. N. Ural'tseva, Linear and quasilinear elliptic equations, Math. Sci. Eng. 46, Academic Press, New York, 1968. MR 39 \#5941 Zbl 0164.13002

[Lieberman 1991] G. M. Lieberman, "The natural generalization of the natural conditions of Ladyzhenskaya and Ural'tseva for elliptic equations", Comm. Partial Differential Equations 16:2-3 (1991), 311-361. MR 92c:35041 Zbl 0742.35028

[Pucci and Serrin 2007] P. Pucci and J. Serrin, The maximum principle, Progr. Nonlinear Differential Eqns. Appl. 73, Birkhäuser, Basel, 2007. MR 2008m:35001 Zbl 1134.35001

[Sun 2012] M. Sun, "Multiplicity of solutions for a class of the quasilinear elliptic equations at resonance”, J. Math. Anal. Appl. 386:2 (2012), 661-668. MR 2012h:35122 Zbl 1229.35089

[Vázquez 1984] J. L. Vázquez, "A strong maximum principle for some quasilinear elliptic equations", Appl. Math. Optim. 12:3 (1984), 191-202. MR 86m:35018 Zbl 0561.35003

Received April 27, 2012. Revised January 7, 2013.

Nikolaos S. PAPAgEORgIOU

DEPARTMENT OF MATHEMATICS

NATIONAL TECHNICAL UNIVERSITY OF ATHENS

ZOGRAFOU CAMPUS

15780 ATHENS

GREECE

npapg@math.ntua.gr

GEORGE SMYRLIS

DEPARTMENT OF MATHEMATICS

National TECHNiCAl University OF ATHENS

ZOGRAFOU CAMPUS

15780 ATHENS

GREECE

gsmyrlis@math.ntua.gr 


\title{
PACIFIC JOURNAL OF MATHEMATICS
}

\author{
msp.org/pjm
}

Founded in 1951 by E. F. Beckenbach (1906-1982) and F. Wolf (1904-1989)

\section{EDITORS}

V. S. Varadarajan (Managing Editor)

Department of Mathematics

University of California

Los Angeles, CA 90095-1555

pacific@math.ucla.edu

Paul Balmer

Department of Mathematics

University of California

Los Angeles, CA 90095-1555

balmer@math.ucla.edu

Daryl Cooper

Department of Mathematics

University of California

Santa Barbara, CA 93106-3080 cooper@math.ucsb.edu

Jiang-Hua $\mathrm{Lu}$

Department of Mathematics

The University of Hong Kong

Pokfulam Rd., Hong Kong jhlu@maths.hku.hk
Don Blasius

Department of Mathematics University of California

Los Angeles, CA 90095-1555

blasius@math.ucla.edu

Robert Finn

Department of Mathematics Stanford University

Stanford, CA 94305-2125

finn@math.stanford.edu

Sorin Popa

Department of Mathematics

University of California

Los Angeles, CA 90095-1555

popa@math.ucla.edu

Paul Yang

Department of Mathematics

Princeton University

Princeton NJ 08544-1000

yang@math.princeton.edu

\section{PRODUCTION}

Silvio Levy, Scientific Editor, production@msp.org

\section{SUPPORTING INSTITUTIONS}

ACADEMIA SINICA, TAIPEI

CALIFORNIA INST. OF TECHNOLOGY

INST. DE MATEMÁTICA PURA E APLICADA

KEIO UNIVERSITY

MATH. SCIENCES RESEARCH INSTITUTE

NEW MEXICO STATE UNIV.

OREGON STATE UNIV.

\author{
STANFORD UNIVERSITY \\ UNIV. OF BRITISH COLUMBIA \\ UNIV. OF CALIFORNIA, BERKELEY \\ UNIV. OF CALIFORNIA, DAVIS \\ UNIV. OF CALIFORNIA, LOS ANGELES \\ UNIV. OF CALIFORNIA, RIVERSIDE \\ UNIV. OF CALIFORNIA, SAN DIEGO \\ UNIV. OF CALIF., SANTA BARBARA
}

\author{
Vyjayanthi Chari \\ Department of Mathematics \\ University of California \\ Riverside, CA 92521-0135 \\ chari@math.ucr.edu \\ Kefeng Liu \\ Department of Mathematics \\ University of California \\ Los Angeles, CA 90095-1555 \\ liu@math.ucla.edu \\ Jie Qing \\ Department of Mathematics \\ University of California \\ Santa Cruz, CA 95064 \\ qing@cats.ucsc.edu
}

These supporting institutions contribute to the cost of publication of this Journal, but they are not owners or publishers and have no responsibility for its contents or policies.

See inside back cover or msp.org/pjm for submission instructions.

The subscription price for 2013 is US \$400/year for the electronic version, and \$485/year for print and electronic.

Subscriptions, requests for back issues and changes of subscribers address should be sent to Pacific Journal of Mathematics, P.O. Box 4163, Berkeley, CA 94704-0163, U.S.A. The Pacific Journal of Mathematics is indexed by Mathematical Reviews, Zentralblatt MATH, PASCAL CNRS Index, Referativnyi Zhurnal, Current Mathematical Publications and the Science Citation Index.

The Pacific Journal of Mathematics (ISSN 0030-8730) at the University of California, c/o Department of Mathematics, 798 Evans Hall \#3840, Berkeley, CA 94720-3840, is published ten to twelve times a year. Periodical rate postage paid at Berkeley, CA 94704, and additional mailing offices. POSTMASTER: send address changes to Pacific Journal of Mathematics, P.O. Box 4163, Berkeley, CA 94704-0163.

PJM peer review and production are managed by EditFLOW ${ }^{\circledR}$ from Mathematical Sciences Publishers.

PUBLISHED BY 


\section{PACIFIC JOURNAL OF MATHEMATICS}

Volume $264 \quad$ No. $2 \quad$ August 2013

On 4-manifolds, folds and cusps

STEFAN BEHRENS

Thin $r$-neighborhoods of embedded geodesics with finite length and

307 negative Jacobi operator are strongly convex

PHILIPPE DELANOË

Eigenvalues of perturbed Laplace operators on compact manifolds

ASMA HASSANNEZHAD

Four equivalent versions of nonabelian gerbes

THOMAS NIKOLAUS and KONRAD WALDORF

On nonlinear nonhomogeneous resonant Dirichlet equations

421

Nikolaos S. PAPAGEORGIOU and GEORGE SMYRLIS

A geometric model of an arbitrary real closed field

455

STANISŁAW SPODZIEJA

Twisted K-theory for the orbifold $[* / G]$

471

MARIO VELÁSQUEZ, EdWARd BECERRA and HERMES

MARTINEZ

Linear restriction estimates for the wave equation with an inverse square potential

JUNYONG ZHANG and JIQIANG ZHENG 